\title{
INDUCED DUSTY FLOW DUE TO NORMAL OSCILLATION OF WAVY WALL
}

\author{
K. KANNAN and V. RAMAMURTHY
}

(Received 22 May 2000)

\begin{abstract}
A two-dimensional viscous dusty flow induced by normal oscillation of a wavy wall for moderately large Reynolds number is studied on the basis of boundary layer theory in the case where the thickness of the boundary layer is larger than the amplitude of the wavy wall. Solutions are obtained in terms of a series expansion with respect to small amplitude by a regular perturbation method. Graphs of velocity components, both for outer flow and inner flow for various values of mass concentration of dust particles are drawn. The inner and outer solutions are matched by the matching process. An interested application of present result to mechanical engineering may be the possibility of the fluid and dust transportation without an external pressure.
\end{abstract}

2000 Mathematics Subject Classification. 76Txx.

1. Introduction. The study of fluids flow induced by unsteady motion of a wall is of great practical importance in the field of biophysics. The problems of the flow of fluids induced by the sinusoidal wavy motion of a wall have been discussed by Taylor [6], Burns and Parkes [1], Yin and Fung [7], and Tanaka [5]. Tanaka studied the problem both for small and moderately large Reynolds number. While studying the problem for moderately large Reynolds numbers, he has shown that if the thickness of the boundary layer is larger than the amplitude of the wavy wall, the technique employed for small Reynolds numbers can also be applied to the case of moderately large Reynolds numbers.

Recently, while studying the flow of blood through mammalian capillaries, blood is taken to be a binary system of plasma (liquid phase) and blood cells (solid phase). In order to gain some insight into the peristaltic motion of blood in capillaries, it is of interest to study the induced flow of a dusty or two-phase fluid by sinusoidal motion of a wavy wall.

Tanaka [5] discussed a two-dimensional flow of an incompressible viscous fluid due to an infinite sinusoidal wavy wall which executes progressive motion with constant speed. In 1980, S. K. Nag extended the same problem for a dusty fluid, up to secondorder solution. Ramamurthy and Rao [4] studied the two-dimensional flow of a dusty fluid which is an extension of Tanaka's work. Dhar and Nandha [2] studied the motion of the two-dimensional fluid with the motion of the wall being described as a nonprogressive wave $(y=a \cos (2 \pi x / L) \sin \omega t)$ up to third-order solution. In the present paper, we have studied the two-dimensional flow of an incompressible dusty fluid, by taking the motion of the wall as a nonprogressive wave (as in Dhar's work) and discussed the problem up to fourth-order solution. The effect of dust parameter on 
the entire flow field has been classified more elaborately by taking up the fourth-order solutions for discussion. Solutions are obtained in terms of series expansion with respect to the small amplitude by regular perturbation method. The inner (boundary layer flow) and the outer (flow beyond the boundary layer), solutions are matched by a matching process given by Kevorkian and Cole [3]. Graphs of the velocity components, both for the outer and the inner flow for various values of mass-concentration of the dust particles are drawn.

2. Formulation of the problem. In order to formulate the fundamental equations of motion of the two-phase fluid flows and to bring out the essential features, certain basic assumptions are made. They are as follows:

(1) The fluid is an incompressible Newtonian fluid.

(2) Dust particles are assumed to be spherical in shape, all having the same radius and mass and undeformable.

(3) The bulk concentration (i.e., concentration by volume) of the dust is very small so that the net effect of the dust on the fluid particles is equivalent to an extra force $\alpha \lambda\left(\vec{q}_{p}-\vec{q}\right)$ per unit volume, (given by P. G. Saffman), where $\vec{q}(x, t)$ is the velocity vector of the fluid, $\vec{q}_{p}(x, t)$ is the velocity vector of the dust particles.

(4) It is also assumed that the Reynolds number of the relative motion of the dust and the fluid is small compared with unity.

The effect of the dust enters through the two parameters $\alpha$ and $\lambda$.

We consider a two-dimensional flow of an incompressible viscous dusty fluid due to an infinite sinusoidal wavy wall of amplitude $a$ and wave length $L$, which is oscillating vertically with a frequency $\omega / 2 \pi$ and an amplitude $a \cos (2 \pi x / L), x$ being the coordinate in the down stream direction of the flow and $y$, the coordinate perpendicular to it. The motion of the wall is described by

$$
y=h(x, t)=a \cos \left(\frac{2 \pi x}{L}\right) \sin \omega t,
$$

where $a$ is the amplitude of the wavy wall, $L$ is the wave length, and $\omega$ is the frequency. The equations of conservation of momentum for the fluid and the dust are given by

$$
\begin{gathered}
\rho\left(\frac{\partial \vec{q}}{\partial t}+(\vec{q} \cdot \nabla) \vec{q}\right)=-\nabla p+\mu\left(\nabla^{2} \cdot \vec{q}\right)+K N_{o}\left(\vec{q}_{p}-\vec{q}\right), \\
N_{\mathrm{o}} m\left(\frac{\partial \vec{q}_{p}}{\partial t}+\left(\vec{q}_{p} \cdot \nabla\right) \vec{q}_{p}\right)=K N_{\mathrm{o}}\left(\vec{q}-\vec{q}_{p}\right),
\end{gathered}
$$

where $p$ is fluid pressure, $K$ is Stokes resistant coefficient, $m$ is the mass of a particle $N_{\mathrm{o}}$ is the number density and $\mu$ is the coefficient of viscosity. Here we assume that $(a / L) \ll 1$.

We normalize all lengths by characteristic length $(L / 2 \pi)$, all velocities $\left\{\vec{q}\right.$ and $\left.\vec{q}_{p}\right\}$ by characteristic speed $(L \omega / 2 \pi)$, the fluid pressure $p$ by $\left(\rho L^{2} \omega^{2} / 4 \pi^{2}\right)$, the time by characteristic time $(1 / \omega)$. The above equations of motion of the fluid and dust become

$$
\vec{q}_{t}+(\vec{q} \cdot \nabla) \vec{q}=-\operatorname{grad} \cdot p+\left(\frac{1}{R}\right) \nabla^{2} \vec{q}+\alpha \lambda\left(\vec{q}_{p}-\vec{q}\right),
$$




$$
\begin{gathered}
\left(\vec{q}_{p}\right)_{t}+\left(\vec{q}_{p} \cdot \nabla\right) \vec{q}_{p}=\alpha\left(\vec{q}-\vec{q}_{p}\right), \\
\operatorname{div} \vec{q}=0, \quad \operatorname{div} \vec{q}_{p}=0,
\end{gathered}
$$

where the nondimensional parameters concerning the dust are

$$
\lambda=\frac{m N_{\mathrm{o}}}{L}, \quad \alpha=\frac{K L}{2 \pi c m},
$$

and Reynolds number $R=\left(L^{2} \omega / 4 \pi^{2} v\right), K=6 \pi \mu a$, where $c$ is the characteristic speed, $v$ is the kinematic viscosity and $\alpha, \lambda$ are the dust parameters.

The boundary conditions are

$$
\begin{aligned}
& u=0, \quad v=\left(\frac{\partial h}{\partial t}\right) \quad \text { at } y=h(x, t), \\
& |u|,|v|,|u p|,|v p|<\infty \quad \text { as } y \longrightarrow \infty
\end{aligned}
$$

where $h=\varepsilon \cos x \sin t$ and $\varepsilon=(2 \pi \alpha / L) \ll 1$.

Equation (2.7) represents no slip condition of the fluid on the wall.

By introducing the stream function $\Psi(x, y, t)$ and $\Psi_{p}(x, y, t)$ for the fluid dust, respectively, the governing equation (2.3), (2.4), and the boundary condition (2.7), (2.8) become

$$
\begin{gathered}
\frac{\partial}{\partial t}\left(\nabla^{2} \Psi\right)+\frac{\partial \Psi}{\partial y} \nabla^{2}\left(\frac{\partial \Psi}{\partial x}\right)-\frac{\partial \Psi}{\partial x} \nabla^{2}\left(\frac{\partial \Psi}{\partial y}\right)=\frac{1}{R} \nabla^{2}\left(\nabla^{2} \Psi\right)+\lambda \alpha \nabla^{2}\left(\Psi_{p}-\Psi\right) \\
\frac{\partial}{\partial t}\left(\nabla^{2} \Psi_{p}\right)+\frac{\partial \Psi_{p}}{\partial y} \nabla^{2}\left(\frac{\partial \Psi_{p}}{\partial x}\right)-\frac{\partial \Psi_{p}}{\partial x} \nabla^{2}\left(\frac{\partial \Psi}{\partial y}\right)=\alpha \nabla^{2}\left(\Psi-\Psi_{p}\right) \\
\frac{\partial \Psi}{\partial y}=0, \quad-\frac{\partial \Psi}{\partial x}=\frac{\partial h}{\partial t} \quad \text { at } y=h(x, t) \\
\left|\frac{\partial \Psi}{\partial y}\right|,\left|\frac{\partial \Psi}{\partial x}\right|,\left|\frac{\partial \Psi_{p}}{\partial y}\right|,\left|\frac{\partial \Psi_{p}}{\partial x}\right|<\infty \quad \text { as } y \rightarrow \infty
\end{gathered}
$$

3. Solution of the problem. When Reynolds number becomes large, the boundary layer is formed. As we have assumed that the thickness of the boundary layer is larger than the wave amplitude, following Tanaka [5], regular perturbation technique can be applied to the present problem. If $\delta$ is the thickness of the boundary layer, the nondimensional may be defined as $\bar{y}=(y / \delta)$ and $\bar{\Psi}=(\Psi / \delta)$. When the viscous term is supposed to be of the same order as the inertia terms, we have that $\delta^{2} R$ is $0(1)$ as usual. The boundary conditions at $y=h$ are expanded into Taylor series around $h=0$ in terms of the inner variables $\bar{\Psi}$ and $\bar{y}$ as

$$
\begin{gathered}
\frac{\partial \bar{\Psi}}{\partial x}(0)+\frac{h}{\delta} \frac{\partial^{2} \bar{\Psi}}{\partial x \partial \bar{y}}(0)+\frac{1}{2 !} \frac{h^{2}}{\delta^{2}} \frac{\partial^{3} \bar{\Psi}}{\partial x \partial \bar{y}^{2}}(0)+\cdots=-\frac{1}{\delta} \frac{\partial h}{\partial t}, \\
\frac{\partial \bar{\Psi}}{\partial \bar{y}}(0)+\frac{h}{\delta} \frac{\partial^{2} \bar{\Psi}}{\partial \bar{y}^{2}}(0)+\frac{1}{2 !} \frac{h^{2}}{\delta^{2}} \frac{\partial^{3} \bar{\Psi}}{\partial \bar{y}^{3}}(0)+\cdots=0 .
\end{gathered}
$$

In order that Taylor series converges, $0(\delta)$ must be larger than $0(h)$, that is, $0(\varepsilon)<$ $0(\delta)$. Following Tanaka [5], we take $\delta=r \varepsilon^{1 / 2}, r$ being an arbitrary constant of $0(1)$. 
The outer flow (the flow beyond the boundary layer) is described by (2.9) in terms of the original variables $\left(\Psi, \Psi_{p}, x, y, t\right)$ while the inner flow (boundary layer flow) is described in terms of the inner variables $\left(\bar{\Psi}, \bar{\Psi}_{p}, x, \bar{y}, t\right)$ on substituting $R=\left(r^{2} \varepsilon\right)^{-1}$ and $\delta=r \varepsilon^{1 / 2}$. As $\varepsilon \ll 1$, we can use perturbation method and assume that $\Psi$, $\Psi_{p}$ (outer flow) and $\bar{\Psi}, \bar{\Psi}_{p}$ (inner flow) can be expanded as power series in $\varepsilon^{1 / 2}$ using

$$
\left(\Psi, \Psi_{p}, \bar{\Psi}, \bar{\Psi}_{p}\right)=\sum_{n=1}^{\infty} \varepsilon^{n / 2}\left(\Psi_{n},\left(\Psi_{p}\right)_{n}, \bar{\Psi}_{n},\left(\bar{\Psi}_{p}\right)_{n}\right) .
$$

Substituting (3.2) and using $\bar{y}=(y / \delta), \bar{\Psi}=(\Psi / \delta), R=\left(r^{2} \varepsilon\right)^{-1}, \delta=r \varepsilon^{1 / 2}$ in (2.9), (2.10), and the boundary conditions (3.1) and then equating the coefficients of like power of $\varepsilon^{1 / 2}$ and by using the following differential operators,

$$
\begin{gathered}
L[\Psi]=-\frac{\partial}{\partial t} \nabla^{2} \Psi, \quad L\left(\Psi_{p}\right)=-\frac{\partial}{\partial t} \nabla^{2} \Psi_{p}, \\
M[\bar{\Psi}]=\left(\frac{\partial^{4}}{\partial \bar{y}^{4}}-\frac{\partial^{3}}{\partial t \partial \bar{y}^{2}}\right) \bar{\Psi}, \quad M\left(\bar{\Psi}_{p}\right)=-\frac{\partial^{3}}{\partial t \partial \bar{y}^{2}}\left[\bar{\Psi}_{p}\right] .
\end{gathered}
$$

We obtain the equation and the boundary conditions corresponding to first order, second order, etc., as follows.

FIRST ORDER $\left(\left[0\left(\varepsilon^{1 / 2}\right)\right]\right)$

Outer:

$$
\begin{aligned}
L\left[\Psi_{1}\right] & =-\alpha \lambda \nabla^{2}\left(\Psi_{p^{1}}-\Psi_{1}\right), \\
L\left[\Psi_{p^{1}}\right] & =-\alpha\left(\Psi_{1}-\Psi_{p}\right) .
\end{aligned}
$$

Inner:

$$
\begin{gathered}
M\left(\Psi_{1}\right)=-\alpha \lambda \frac{\partial^{2}}{\partial y^{2}}\left(\bar{\Psi}_{p^{1}}-\bar{\Psi}_{1}\right) \\
M\left(\Psi_{p^{1}}\right)=-\alpha \frac{\partial^{2}}{\partial y^{2}}\left(\bar{\Psi}_{1}-\bar{\Psi}_{p}\right) \\
\frac{\partial \bar{\Psi}_{1}}{\partial \bar{y}}(0)=0, \quad \frac{\partial \bar{\Psi}_{1}}{\partial x}=-\frac{\cos x \cos t}{r} .
\end{gathered}
$$

SECOND ORDER $(0(\varepsilon))$

Outer:

$$
\begin{aligned}
L\left[\Psi_{2}\right]+\alpha \lambda \nabla^{2}\left(\Psi_{p^{2}}-\Psi_{2}\right) & =\frac{\partial \Psi_{1}}{\partial y} \nabla^{2} \frac{\partial \Psi_{1}}{\partial x}-\frac{\partial \Psi_{1}}{\partial x} \nabla^{2} \frac{\partial \Psi_{1}}{\partial y} \\
L\left[\Psi_{p^{2}}\right]+\alpha \nabla^{2}\left(\Psi_{2}-\Psi_{p^{2}}\right) & =\frac{\partial \Psi_{p^{1}}}{\partial y} \nabla^{2} \frac{\partial \Psi_{p^{1}}}{\partial x}-\frac{\partial \Psi_{p^{1}}}{\partial x} \nabla^{2} \frac{\partial \Psi_{p^{1}}}{\partial y} .
\end{aligned}
$$

Inner:

$$
\begin{gathered}
M\left(\bar{\Psi}_{2}\right)+\alpha \lambda \frac{\partial^{2}}{\partial \bar{y}^{2}}\left(\bar{\Psi}_{p^{2}}-\bar{\Psi}_{2}\right)=\frac{\partial \bar{\Psi}_{1}}{\partial \bar{y}} \frac{\partial^{3} \bar{\Psi}_{1}}{\partial x \partial \bar{y}^{2}}-\frac{\partial \bar{\Psi}_{1}}{\partial x} \frac{\partial^{3} \bar{\Psi}_{1}}{\partial \bar{y}^{3}} \\
M\left(\bar{\Psi}_{p^{2}}\right)+\alpha \frac{\partial^{2}}{\partial \bar{y}^{2}}\left(\bar{\Psi}_{2}-\bar{\Psi}_{p^{2}}\right)=\frac{\partial \bar{\Psi}_{p^{1}}}{\partial \bar{y}} \cdot \frac{\partial^{3} \bar{\Psi}_{p^{1}}}{\partial x \partial \bar{y}^{2}}-\frac{\partial \bar{\Psi}_{p^{1}}}{\partial x} \frac{\partial^{3} \bar{\Psi}_{p^{1}}}{\partial \bar{y}^{3}} \\
\frac{\partial \bar{\Psi}_{2}}{\partial \bar{y}}(0)=-\frac{\cos x \sin t}{r} \frac{\partial^{2} \bar{\Psi}_{1}}{\partial \bar{y}^{2}}(0), \quad \frac{\partial \bar{\Psi}_{2}}{\partial x}(0)=-\frac{\cos x \sin t}{r} \frac{\partial^{2} \bar{\Psi}_{1}}{\partial x \partial \bar{y}}(0)
\end{gathered}
$$


THIRD ORDER $\left(0\left(\varepsilon^{3 / 2}\right)\right)$

Outer:

$$
\begin{aligned}
L\left(\Psi_{3}\right)+\alpha \lambda \nabla^{2}\left(\Psi_{p^{3}}-\Psi_{3}\right)= & -r^{2} \nabla^{2} \nabla^{2} \Psi_{1}+\frac{\partial \Psi_{1}}{\partial y} \nabla^{2} \frac{\partial \Psi_{2}}{\partial x} \\
& +\frac{\partial \Psi_{2}}{\partial y} \nabla^{2} \frac{\partial \Psi_{1}}{\partial x}-\frac{\partial \Psi_{1}}{\partial x} \nabla^{2} \frac{\partial \Psi_{2}}{\partial y}-\frac{\partial \Psi_{2}}{\partial x} \nabla^{2} \frac{\partial \Psi_{1}}{\partial y}, \\
L\left(\Psi_{p^{3}}\right)+\alpha \nabla^{2}\left(\Psi_{3}-\Psi_{p^{3}}\right)= & -r^{2} \nabla^{2} \nabla^{2} \Psi_{p^{1}}+\frac{\partial \Psi_{p^{1}}}{\partial y} \nabla^{2} \frac{\partial \Psi_{p^{2}}}{\partial x} \\
& +\frac{\partial \Psi_{p^{2}}}{\partial y} \nabla^{2} \frac{\partial \Psi_{p^{1}}}{\partial x}-\frac{\partial \Psi_{p^{1}}}{\partial x} \nabla^{2} \frac{\partial \Psi_{p^{2}}}{\partial y}-\frac{\partial \Psi_{p^{2}}}{\partial x} \nabla^{2} \frac{\partial \Psi_{p^{1}}}{\partial y} .
\end{aligned}
$$

Inner:

$$
\begin{gathered}
M\left(\bar{\Psi}_{3}\right)+\alpha \lambda\left(r^{2} \frac{\partial^{2}}{\partial x^{2}}\left(\bar{\Psi}_{p^{1}}-\bar{\Psi}_{1}\right)+\frac{\partial^{2}}{\partial \bar{y}^{2}}\left(\bar{\Psi}_{p^{3}}-\bar{\Psi}_{3}\right)\right) \\
=-2 r^{2} \frac{\partial^{4}}{\partial x^{2}} \frac{\bar{\Psi}_{1}}{\partial \bar{y}^{2}}+r^{2} \frac{\partial^{3} \bar{\Psi}_{1}}{\partial t \partial x^{2}}+\frac{\partial \bar{\Psi}_{1}}{\partial \bar{y}} \frac{\partial^{3} \bar{\Psi}_{2}}{\partial x \partial \bar{y}^{2}}+\frac{\partial \bar{\Psi}_{2}}{\partial \bar{y}} \cdot \frac{\partial^{3} \bar{\Psi}_{1}}{\partial x \partial \bar{y}^{2}}-\frac{\partial \bar{\Psi}_{1}}{\partial x} \cdot \frac{\partial^{3} \bar{\Psi}_{2}}{\partial \bar{y}^{3}}-\frac{\partial \bar{\Psi}_{2}}{\partial x} \cdot \frac{\partial^{3} \bar{\Psi}_{1}}{\partial \bar{y}_{3}}, \\
M\left(\bar{\Psi}_{p^{3}}\right)+\alpha\left(r^{2} \frac{\partial^{2}}{\partial x^{2}}\left(\bar{\Psi}_{1}-\bar{\Psi}_{p^{1}}\right)+\frac{\partial^{2}}{\partial \bar{y}^{2}}\left(\bar{\Psi}_{3}-\bar{\Psi}_{p^{3}}\right)\right) \\
=-2 r^{2} \frac{\partial^{4}}{\partial x^{2}} \frac{\bar{\Psi}_{p^{1}}}{\partial \bar{y}^{2}}+r^{2} \frac{\partial^{3} \bar{\Psi}_{p^{1}}}{\partial t \partial x^{2}}+\frac{\partial \bar{\Psi}_{1}}{\partial \bar{y}} \frac{\partial^{3} \bar{\Psi}_{2}}{\partial x \partial \bar{y}^{2}}+\frac{\partial \bar{\Psi}_{p^{2}}}{\partial \bar{y}} \cdot \frac{\partial^{3} \bar{\Psi}_{1}}{\partial x \partial \bar{y}^{2}}-\frac{\partial \bar{\Psi}_{p^{1}}}{\partial x} \cdot \frac{\partial^{3} \bar{\Psi}_{p^{2}}}{\partial \bar{y}^{3}}-\frac{\partial \bar{\Psi}_{p^{2}}}{\partial x} \cdot \frac{\partial^{2} \bar{\Psi}_{p^{1}}}{\partial \bar{y}^{2}}, \\
\frac{\partial \bar{\Psi}_{3}}{\partial \bar{y}}(0)=-\frac{1}{r} \cos x \sin t \frac{\partial^{2} \bar{\Psi}_{2}}{\partial \bar{y}^{2}}(0)-\frac{1}{2 r^{2}} \cos ^{2} x \sin ^{2} t \frac{\partial^{3} \bar{\Psi}_{1}}{\partial \bar{y}^{3}}(0), \\
\frac{\partial \bar{\Psi}_{3}}{\partial x}(0)=-\frac{1}{r} \cos x \sin t \frac{\partial^{2} \bar{\Psi}_{2}}{\partial x \partial \bar{y}}(0)-\frac{1}{2 r^{2}} \cos ^{2} x \sin ^{2} t \frac{\partial^{3} \bar{\Psi}_{1}}{\partial x \partial \bar{y}^{2}}(0) .
\end{gathered}
$$

FOURTH ORDER $\left(0\left(\varepsilon^{2}\right)\right)$

Outer:

$$
\begin{aligned}
L\left[\Psi_{4}\right]+\alpha \lambda \nabla^{2}\left(\Psi_{p^{4}}-\Psi_{4}\right)= & -r^{2} \nabla^{2} \nabla^{2} \Psi_{2}+\frac{\partial \Psi_{1}}{\partial y} \nabla^{2} \frac{\partial \Psi_{3}}{\partial x}+\frac{\partial \Psi_{2}}{\partial y} \nabla^{2} \frac{\partial \Psi_{2}}{\partial x} \\
& +\frac{\partial \Psi_{3}}{\partial y} \nabla^{2} \frac{\partial \Psi_{1}}{\partial x}-\frac{\partial \Psi_{1}}{\partial x} \nabla^{2} \frac{\partial \Psi_{3}}{\partial y}-\frac{\partial \Psi_{2}}{\partial x} \nabla^{2} \frac{\partial \Psi_{2}}{\partial y}-\frac{\partial \Psi_{3}}{\partial x} \nabla^{2} \frac{\partial \Psi_{1}}{\partial y} \\
L\left[\Psi_{p^{4}}\right]+\alpha \lambda \nabla^{2}\left(\Psi_{4}-\Psi_{p^{4}}\right)= & \frac{\partial \Psi_{p^{1}}}{\partial y} \nabla^{2} \frac{\partial \Psi_{p^{3}}}{\partial x}+\frac{\partial \Psi_{p^{2}}}{\partial y} \nabla^{2} \frac{\partial \Psi_{p^{2}}}{\partial x}+\frac{\partial \Psi_{p^{3}}}{\partial y} \nabla^{2} \frac{\partial \Psi_{p^{1}}}{\partial x} \\
& -\frac{\partial \Psi_{p^{1}}}{\partial x} \nabla^{2} \frac{\partial \Psi_{p^{3}}}{\partial y}-\frac{\partial \Psi_{p^{2}}}{\partial x} \nabla^{2} \frac{\partial \Psi_{p^{2}}}{\partial y}-\frac{\partial \Psi_{p^{3}}}{\partial x} \nabla^{2} \frac{\partial \Psi_{p^{1}}}{\partial y}
\end{aligned}
$$


Inner:

$$
\begin{aligned}
& M\left(\bar{\Psi}_{4}\right)+\alpha \lambda\left(r^{2} \frac{\partial^{2}}{\partial x^{2}}\left(\bar{\Psi}_{p^{2}}-\bar{\Psi}_{2}\right)+\frac{\partial^{2}}{\partial \bar{y}^{2}}\left(\bar{\Psi}_{p^{4}}-\bar{\Psi}_{4}\right)\right) \\
& =-2 r^{2} \frac{\partial^{4}}{\partial x^{2}} \frac{\bar{\Psi}_{2}}{\partial \bar{y}^{2}}+r^{2} \frac{\partial^{3} \bar{\Psi}_{2}}{\partial t \partial x^{2}}+r^{2} \frac{\partial \bar{\Psi}_{1}}{\partial \bar{y}} \frac{\partial^{3} \bar{\Psi}_{1}}{\partial x^{3}}-r^{2} \frac{\partial \bar{\Psi}_{1}}{\partial x} \frac{\partial^{3} \bar{\Psi}_{1}}{\partial x^{2} \partial \bar{y}} \\
& +\frac{\partial \bar{\Psi}_{1}}{\partial \bar{y}} \cdot \frac{\partial^{3} \bar{\Psi}_{3}}{\partial x \partial \bar{y}^{2}}+\frac{\partial \bar{\Psi}_{2}}{\partial \bar{y}} \frac{\partial^{3} \bar{\Psi}_{2}}{\partial x \partial \bar{y}^{2}}+\frac{\partial \bar{\Psi}_{3}}{\partial \bar{y}} \frac{\partial^{3} \bar{\Psi}_{1}}{\partial x \partial \bar{y}^{2}}-\frac{\partial \bar{\Psi}_{1}}{\partial x} \cdot \frac{\partial^{3} \bar{\Psi}_{3}}{\partial \bar{y}^{3}}-\frac{\partial \bar{\Psi}_{2}}{\partial x} \cdot \frac{\partial^{3} \bar{\Psi}_{2}}{\partial \bar{y}^{3}}-\frac{\partial \bar{\Psi}_{3}}{\partial x} \frac{\partial^{3} \bar{\Psi}_{1}}{\partial \bar{y}^{3}}, \\
& M\left(\bar{\Psi}_{p^{4}}\right)+\alpha\left(r^{2} \frac{\partial^{2}}{\partial x^{2}}\left(\bar{\Psi}_{2}-\bar{\Psi}_{p^{2}}\right)+\frac{\partial^{2}}{\partial \bar{y}^{2}}\left(\bar{\Psi}_{4}-\bar{\Psi}_{p^{4}}\right)\right) \\
& =r^{2} \frac{\partial^{3} \bar{\Psi}_{p^{2}}}{\partial t \partial x^{2}}+r^{2} \frac{\partial \bar{\Psi}_{p^{1}}}{\partial \bar{y}} \frac{\partial^{3} \bar{\Psi}_{p^{1}}}{\partial x^{3}}-r^{2} \frac{\partial \bar{\Psi}_{p^{1}}}{\partial x} \frac{\partial^{3} \bar{\Psi}_{p^{1}}}{\partial x^{2} \partial \bar{y}}+\frac{\partial \bar{\Psi}_{p^{1}}}{\partial \bar{y}} \cdot \frac{\partial^{3} \bar{\Psi}_{p^{3}}}{\partial x \partial \bar{y}^{2}} \\
& +\frac{\partial \bar{\Psi}_{p^{2}}}{\partial \bar{y}} \frac{\partial^{3} \bar{\Psi}_{p^{2}}}{\partial x \partial \bar{y}^{2}}+\frac{\partial \bar{\Psi}_{p^{3}}}{\partial \bar{y}} \cdot \frac{\partial^{3} \bar{\Psi}_{p^{1}}}{\partial x \partial \bar{y}^{2}}-\frac{\partial \bar{\Psi}_{p^{1}}}{\partial x} \cdot \frac{\partial^{3} \bar{\Psi}_{p^{3}}}{\partial \bar{y}^{3}}-\frac{\partial \bar{\Psi}_{p^{2}}}{\partial x} \cdot \frac{\partial^{3} \bar{\Psi}_{p^{2}}}{\partial \bar{y}^{3}}-\frac{\partial \bar{\Psi}_{p^{3}}}{\partial x} \frac{\partial^{3} \bar{\Psi}_{p^{1}}}{\partial \bar{y}^{3}}, \\
& \frac{\partial \bar{\Psi}_{4}}{\partial \bar{y}}(0)=-\frac{1}{r} \cos x \sin t \frac{\partial^{2} \bar{\Psi}_{3}}{\partial \bar{y}^{2}}(0)-\frac{1}{2 r^{2}} \cos ^{2} x \cdot \sin ^{2} t \frac{\partial^{3} \bar{\Psi}_{2}}{\partial \bar{y}^{3}}(0) \\
& -\frac{1}{6 r^{3}} \cos ^{3} x \sin ^{3} t \cdot \frac{\partial^{4} \bar{\Psi}_{1}}{\partial \bar{y}^{4}}(0), \\
& \frac{\partial \bar{\Psi}_{4}}{\partial x}(0)=-\frac{1}{r} \cos x \sin t \frac{\partial^{2} \bar{\Psi}_{3}}{\partial \bar{y}^{2}}(0)-\frac{1}{2 r^{2}} \cos ^{2} x \cdot \sin ^{2} t \frac{\partial^{3} \bar{\Psi}_{2}}{\partial x \partial \bar{y}^{2}}(0) \\
& -\frac{1}{6 r^{3}} \cos ^{3} x \sin ^{3} t \cdot \frac{\partial^{4} \bar{\Psi}_{1}}{\partial x \partial \bar{y}^{3}}(0) \text {. }
\end{aligned}
$$

A series of the inner solutions should satisfy the boundary conditions on the wall, while the outer solution are restricted to be bounded as $y$ increases, that is,

$$
\left|\frac{\partial \Psi_{n}}{\partial x}\right|,\left|\frac{\partial \Psi_{n}}{\partial y}\right|<\infty, \quad\left|\frac{\partial \Psi_{p^{n}}}{\partial x}\right|,\left|\frac{\partial \Psi_{p^{n}}}{\partial y}\right|<\infty
$$

as $y \rightarrow \infty$, for $n=1,2,3 \ldots$.

It is necessary to match the inner and outer solutions. Following Keverkian and Cole [3], the matching is carried out for both $x$ and $y$ components of velocity of fluid and dust by the following principles:

$$
\begin{gathered}
L t \frac{1}{\varepsilon^{N / 2}}\left(\sum_{n=1}^{N} \varepsilon^{n / 2} \frac{\partial \Psi_{n}}{\partial y}-\sum_{n=1}^{N} \varepsilon^{n / 2} \frac{\partial \bar{\Psi}_{n}}{\partial \bar{y}}\right)=0, \\
L t \frac{1}{\varepsilon^{N / 2}}\left(\sum_{n=1}^{N} \varepsilon^{n / 2} \frac{\partial \Psi_{p^{n}}}{\partial y}-\sum_{n=1}^{N} \varepsilon^{n / 2} \frac{\partial \bar{\Psi}_{p^{n}}}{\partial \bar{y}}\right)=0, \quad \varepsilon \rightarrow 0, \bar{y} \text { fixed, }
\end{gathered}
$$




$$
\begin{gathered}
L t \frac{1}{\varepsilon^{N / 2}}\left(\sum_{n=1}^{N} \varepsilon^{n / 2} \frac{\partial \Psi_{n}}{\partial x}-r \varepsilon^{1 / 2} \sum_{n=1}^{N} \varepsilon^{n / 2} \frac{\partial \bar{\Psi}_{n}}{\partial x}\right)=0, \\
L t \frac{1}{\varepsilon^{N / 2}}\left(\sum_{n=1}^{N} \varepsilon^{n / 2} \frac{\partial \Psi_{p^{n}}}{\partial x}-r \varepsilon^{1 / 2} \sum_{n=1}^{N} \varepsilon^{n / 2} \frac{\partial \bar{\Psi}_{p^{n}}}{\partial x}\right)=0, \quad \varepsilon \rightarrow 0, \bar{y} \text { fixed, }
\end{gathered}
$$

up to $N$ th order of magnitude.

We seek solutions of first order in the following form:

$$
\begin{gathered}
\bar{\Psi}_{1}(x, \bar{y}, t)=\sin x\left[F_{1}(\bar{y}) e^{i t}+F_{1}^{*}(\bar{y}) e^{-i t}\right]+F_{1 s}(\bar{y}), \\
\bar{\Psi}_{p^{1}}(x, \bar{y}, t)=\sin x\left[F_{p^{1}}(\bar{y}) e^{i t}+F_{p^{1}} *(\bar{y}) e^{-i t}\right]+F_{p^{1 s}}(\bar{y}), \\
\Psi_{1}(x, y, t)=\sin x\left[f_{1}(y) e^{i t}+f_{1}^{*}(y) e^{-i t}\right]+f_{1 s}(y), \\
\bar{\Psi}_{p^{1}}(x, y, t)=\sin x\left[f_{p^{1}}(y) e^{i t}+f_{p^{1}}^{*}(y) e^{-i t}\right]+F_{p^{1 s}}(y),
\end{gathered}
$$

where $*$ denotes complex conjugation. By substituting (3.26) in the first-order differential equations (3.4), (3.6), and the boundary condition (3.7), we obtain the following system of equations:

$$
\begin{gathered}
\frac{d^{4} F_{1}}{d \bar{y}^{4}}-i \frac{d^{2} F_{1}}{d \bar{y}^{2}}=-\alpha \lambda\left(\frac{d^{2} F_{p^{1}}}{d \bar{y}^{2}}-\frac{d^{2} F_{1}}{d \bar{y}^{2}}\right), \quad \frac{d^{4} F_{p^{1 s}}}{d \bar{y}^{4}}=-\alpha \lambda\left(\frac{d^{2} F_{p^{1 s}}}{d \bar{y}^{2}}-\frac{d^{2} F_{1 s}}{d \bar{y}^{2}}\right), \\
\frac{d^{2} F_{p^{1}}}{d \bar{y}^{2}}=\frac{\alpha}{\alpha+i} \frac{d^{2} F_{1}}{d \bar{y}^{2}}, \quad \frac{d^{2} F_{p^{1 s}}}{d \bar{y}^{2}}=\frac{d^{2} F_{1 s}}{d \bar{y}^{2}}, \quad \frac{d^{2} f_{1}}{d y^{2}}-f_{1}=0, \\
\frac{d^{2} f_{p^{1}}}{d y^{2}}-f_{p^{1}}=\frac{\alpha}{\alpha+i}\left(\frac{d^{2} f_{1}}{d y^{2}}-f_{1}\right), \\
\frac{d^{2} f_{1 s}}{d y^{2}}=\frac{d^{2} f_{p^{1 s}}}{d y^{2}},
\end{gathered}
$$

and their solutions are

$$
\begin{gathered}
F_{1}=A_{1} e^{-\lambda_{1} \beta \bar{y}}-\frac{1}{2 r}+\lambda_{1} \beta A_{1} \bar{y}-A_{1}, \\
F_{p^{1}}=\frac{\alpha}{\alpha+i}\left[F_{1}\right]+A \bar{y}, \quad \frac{d F_{p^{1}}}{d \bar{y}}=\frac{d F_{1 s}}{d \bar{y}}=B_{1} \bar{y}+B_{2} \bar{y}^{2}, \\
f_{1}=a_{1} e^{-y}, \quad f_{p^{1}}=a_{2} e^{-y} .
\end{gathered}
$$

When (3.32) are substituted in (3.29) it turns out to be $a_{1}=a_{2}$,

$$
\frac{d f_{1 s}}{d y}=\frac{d f_{p^{1 s}}}{d y}=C_{1}
$$

where

$$
\lambda_{1}=\sqrt{i}=\left(\frac{1+i}{\sqrt{2}}\right), \quad \beta=\frac{\left(Q_{1}-i \lambda \alpha\right)^{1 / 2}}{\sqrt{\alpha^{2}+1}},
$$

$Q_{1}=\left[\alpha^{2}(\lambda+1)+1\right]$ and $A_{1}, B_{1}, B_{2}, a_{1}$, and $C_{1}$ are constants. 
Substituting (3.31), (3.32), and (3.33) into (3.19) and (3.20), we have

$$
\begin{aligned}
\lim _{\substack{\varepsilon \rightarrow 0 \\
y \text { fixed }}} \frac{1}{\varepsilon^{1 / 2}}\left(\varepsilon^{1 / 2} \frac{\partial \Psi_{1}}{\partial y}-\varepsilon^{1 / 2} \frac{\partial \bar{\Psi}_{1}}{\partial \bar{y}}\right)= & \lim _{\substack{\varepsilon \rightarrow 0 \\
y}} \sin x\left(-a_{1} e^{-y} e^{i t}+\text { c.c. }\right)+C_{1} \\
& +\varepsilon\left(\sin x\left(-\lambda_{1} \beta A_{1} e^{-\lambda_{1} \beta y}+\lambda_{1} \beta A_{1}\right) e^{i t}+\text { c.c. }\right) \\
& -B_{1} \bar{y}-B_{2} \bar{y}^{2}=0
\end{aligned}
$$

where c.c. stands for the corresponding complex conjugate.

Taking into account that $y=r \varepsilon^{1 / 2} \bar{y}$ expanding the exponential as

$$
e^{-y}=e^{-r \varepsilon^{1 / 2} \bar{y}}=1-r \varepsilon^{1 / 2} \bar{y}+\frac{r^{2}}{2} \varepsilon \bar{y}^{2},
$$

( $\bar{y}$ fixed) and noting that

$$
\exp (-\lambda \bar{y})\left(=\exp \left(-\lambda_{1} \beta \bar{y}\right)=\exp \left(-\frac{\lambda_{1} \beta}{r \varepsilon^{1 / 2}}\right) y, \bar{y} \text { fixed }\right)
$$

decays very rapidly as $\varepsilon \rightarrow 0$ (which is called transcendentally small term (T.S.T.) and is neglected in the matching process).

We have

$$
\begin{aligned}
& \lim _{\substack{\varepsilon \rightarrow 0 \\
\bar{y} \text { fixed }}}\left[\sin x\left(-a_{1}-A_{1} \lambda_{1} \beta\right) e^{i t}+\text { c.c. }+C_{1}-B_{2} \bar{y}^{2}-B_{1} \bar{y}+\text { T.S.T. }+0\left(\varepsilon^{1 / 2}\right)\right]=0, \\
& \lim _{\substack{\varepsilon \rightarrow 0 \\
y \text { fixed }}}\left[\left(-B_{1}+\frac{A_{1} \lambda_{1} \beta \alpha}{\alpha+i}-A\right) \sin x e^{i t}+\text { c.c. }-B_{1} \bar{y}-B_{2} \bar{y}^{2}+\text { T.S.T. }+0\left(\varepsilon^{1 / 2}\right)\right]=0 \text {. }
\end{aligned}
$$

Thus the matching condition is satisfied only if

$$
-a_{1},-A_{1} \lambda_{1} \beta=0, \quad C_{1}=0, \quad-B_{1}+\frac{A_{1} \lambda_{1} \beta \alpha}{\alpha+i}-A=0, \quad B_{1}=B_{2}=C_{1}=0
$$

when similar process is carried for (3.16) we get

$$
\begin{aligned}
& \lim _{\substack{\varepsilon \rightarrow 0 \\
y \text { fixed }}} \frac{1}{\varepsilon^{1 / 2}}\left(\varepsilon^{1 / 2} \frac{\partial \Psi_{1}}{\partial x}-r \varepsilon \frac{\partial \bar{\Psi}_{1}}{\partial x}\right) \\
& =\lim _{\substack{\varepsilon \rightarrow 0 \\
y}} \frac{1}{\varepsilon^{1 / 2}}\left[\varepsilon^{1 / 2} \cos x\left(a_{1} e^{-y} e^{i t}+\text { c.c. }\right)\right. \\
& \left.\quad-r \varepsilon \cos x\left(\text { T.S.T. }+\left(\lambda_{1} \beta A_{1} \bar{y}-\frac{1}{2 r}-A_{1}\right) e^{i t}-(r \varepsilon \cos x)+\text { c.c. }\right)\right]=0,
\end{aligned}
$$




$$
\begin{aligned}
& \lim _{\substack{\varepsilon \rightarrow 0 \\
\bar{y} \text { fixed }}} \frac{1}{\varepsilon^{1 / 2}}\left[\varepsilon^{1 / 2} \cos x\left(a_{1} e^{i t}+\text { c.c. }\right)+0(\varepsilon)\right]=0, \\
& \lim _{\substack{\varepsilon \rightarrow 0 \\
y \text { fixed }}} \frac{1}{\varepsilon^{1 / 2}}\left(\varepsilon^{1 / 2} \frac{\partial \Psi_{p^{1}}}{\partial x}-r \varepsilon \frac{\partial \bar{\Psi}_{p^{1}}}{\partial x}\right) \\
& =\lim _{\substack{\varepsilon \rightarrow 0 \\
y}} \frac{1}{\varepsilon^{1 / 2}}\left[\varepsilon^{1 / 2} \cos x\left(a_{1} e^{-y} e^{i t}+\text { c.c. }\right)\right. \\
& \left.\quad-r \varepsilon \cos x\left(\text { T.S.T. }+\left(\left(\frac{\alpha}{\alpha+i}\right) \lambda_{1} \beta A_{1} \bar{y}-\frac{1}{2 r}-A_{1}\right)+A \bar{y}\right) e^{i t}+\text { c.c. }\right]=0, \\
& \lim _{\substack{\varepsilon \rightarrow 0 \\
y}} \frac{1}{\varepsilon^{1 / 2}}\left[\varepsilon^{1 / 2} \cos x\left(a_{1} e^{i t}+\text { c.c. }\right)-0(\varepsilon)\right]=0,
\end{aligned}
$$

so that the matching condition is satisfied if $a_{1}=0$

$$
A_{1}=a_{1}=A+B_{1}+B_{2}=0
$$

The first-order solution is obtained as

$$
\begin{gathered}
\Psi_{1}=0, \quad \Psi_{p^{1}}=0, \quad \bar{\Psi}_{1}=-\frac{1}{2 r}\left(\sin x\left(e^{i t}+e^{-i t}\right)\right)=-\frac{\sin x \cos t}{r}, \\
\bar{\Psi}_{p^{1}}=-\frac{\alpha}{\alpha+i} \frac{1}{2 r} \sin x\left(e^{i t}\right)+\text { c.c. }
\end{gathered}
$$

Next we seek second-order solution $\Psi_{2}, \Psi_{p^{2}}, \bar{\Psi}_{2}$, and $\bar{\Psi}_{p^{2}}$ in the following form:

$$
\begin{gathered}
\bar{\Psi}_{2}=\sin 2 x \cdot F_{2} e^{2 i t}+\sin x F_{21} e^{i t}+\text { c.c. }+F_{2 s}, \\
\bar{\Psi}_{p^{2}}=\sin 2 x \cdot F_{p^{2}} e^{2 i t}+\sin x F_{p^{21}} e^{i t}+\text { c.c. }+F_{p^{2 s}}, \\
\Psi_{2}=\sin 2 x \cdot f_{2} e^{2 i t}+\sin x f_{21} e^{i t}+\text { c.c. }+F_{2 s}, \\
\Psi_{p^{2}}=\sin 2 x \cdot f_{p^{2}} e^{2 i t}+\sin x f_{p^{21}} e^{i t}+\text { c.c. }+F_{p^{2 s}} .
\end{gathered}
$$

Substituting (3.42) into (3.8), (3.9), and (3.11), we get after calculations,

$$
\begin{aligned}
\bar{\Psi}_{2} & =\sin x\left(-\frac{i \lambda_{1}}{2 \beta} e^{-\lambda_{1} \beta \bar{y}}+\frac{\bar{y}}{2}+\frac{i \lambda_{1}}{2 \beta}\right) e^{i t}+\text { c.c., } \\
\bar{\Psi}_{p^{2}} & =\frac{\alpha}{\alpha+i}\left(-\frac{i \lambda_{1}}{2 \beta} e^{-\lambda_{1} \beta \bar{y}}+\frac{\bar{y}}{2}+\frac{i \lambda_{1}}{2 \beta}\right) \sin x \cdot e^{i t}+\text { c.c. } \\
\Psi_{2} & =\sin x\left(-\frac{e^{-y}}{2} e^{i t}\right)+\text { c.c. } \\
\Psi_{p^{2}} & =\left(-\frac{\alpha}{\alpha+i}\left(-\left(\frac{\sin x}{2}\right) e^{-y}\right)\right) e^{i t}+\text { c.c. }
\end{aligned}
$$


We seek third-order solutions in the form

$$
\begin{gathered}
\bar{\Psi}_{3}=\sin 3 x F_{3} e^{3 i t}+\sin 2 x F_{32} e^{2 i t}+\sin x F_{31} e^{i t}+\text { c.c. }+F_{3 s} \sin 2 x, \\
\bar{\Psi}_{p^{3}}=\sin 3 x F_{p^{3}} e^{3 i t}+\sin 2 x F_{p^{32}} e^{2 i t}+\sin x F_{p^{31}} e^{i t}+\text { c.c. }+F_{3 s} \sin 2 x, \\
\Psi_{3}=\sin 3 x f_{3} e^{3 i t}+\sin 2 x f_{32} e^{2 i t}+\sin x f_{31} e^{i t}+f_{p^{3 s}} \sin 2 x, \\
\Psi_{p^{3}}=\sin 3 x f_{p^{3}} e^{3 i t}+\sin 2 x f_{p^{32}} e^{2 i t}+\sin x f_{p^{31}} e^{i t}+\text { c.c. }+f_{3 s} \sin 2 x,
\end{gathered}
$$

where

$$
\begin{gathered}
F_{3}=0, \quad F_{p^{3}}=0, \quad F_{32}=\frac{i}{8 r} \frac{1}{\beta^{2}-2 \delta^{2}}\left(1-e^{-\lambda_{1} \beta \bar{y}}\right), \\
F_{p^{32}}=F_{32} \frac{\alpha}{\alpha+2 i}+\frac{\alpha}{(\alpha+i)^{2}} \frac{i}{8 r}\left(\lambda_{1}^{2} e^{-\lambda_{1} \beta y}\right), \\
F_{31}=\frac{i r}{2 \beta^{2}}-\frac{i r \lambda_{1}}{2 \beta} y-\frac{i r}{2 \beta^{2}} e^{-\lambda_{1} \beta \bar{y}}-\frac{r}{4} y^{2}, \\
F_{p^{31}}=\frac{\alpha}{\alpha+i} F_{31}, \quad F_{3 s}=\frac{i}{8 r}\left(\frac{\lambda_{1}}{\beta} e^{-\lambda_{1} \beta y}-\frac{\lambda_{1}^{*}}{\beta^{*}} e^{-\lambda_{1} \beta^{*} y}\right), \\
F_{p^{3 s}}=F_{3 s}+\frac{\alpha}{\alpha^{2}+1} \frac{i}{8 r}\left(\frac{\lambda_{1}^{2}}{\beta} e^{-\lambda_{1} \beta \bar{y}}-\frac{\lambda_{1} *^{2}}{\beta^{*}} e^{-\lambda_{1} \beta^{*} \bar{y}}\right),
\end{gathered}
$$

where

$$
\begin{gathered}
\delta=\left(\frac{\left[Q_{2}-2 i \alpha \lambda\right]^{1 / 2}}{\left(\alpha^{2}+4\right)^{1 / 2}}\right), \quad Q_{2}=\alpha^{2}(\lambda+1)+4, \\
f_{3}=0, \quad f_{32}=0, \quad f_{31}=\frac{i r \lambda_{1}}{2 \beta} e^{-y}, \quad f_{3 s}=0, \\
f_{p^{3}}=0, \quad f_{p^{32}}=0, \quad f_{p^{31}}=\frac{\alpha}{\alpha+i} f_{31}, \quad f_{3 p^{s}}=0 .
\end{gathered}
$$

It is to be noted that the third-order inner solutions both for the fluid and dust have a steady streaming components $F_{3 s}$ and $F_{p 3 s}$. However, they attenuate very rapidly as $y$ increases and are confined only in the boundary layer while no steady streaming is induced in the outer layer up to this order of approximation.

Next we seek the fourth-order solution in the following form:

$$
\begin{gathered}
\bar{\Psi}_{4}=\sin 4 x F_{4} e^{4 i t}+\sin 3 x F_{43} e^{3 i t}+\sin 2 x F_{42} e^{2 i t}+\sin x F_{41} e^{i t}+\text { c.c. }+F_{4 s} \sin 2 x, \\
\bar{\Psi}_{p^{4}}=\sin 4 x F_{p^{4}} e^{4 i t}+\sin 3 x F_{p^{43}} e^{3 i t}+\sin 2 x F_{p^{42}} e^{2 i t}+\sin x F_{p^{41}} e^{i t}+\text { c.c. }+F_{p^{4 s}} \sin 2 x, \\
\Psi_{4}=\sin 4 x f_{4} e^{4 i t}+\sin 3 x f_{43} e^{3 i t}+\sin 2 x f_{42} e^{2 i t}+\sin x f_{41} e^{i t}+\text { c.c. }+f_{4 s} \sin 2 x, \\
\Psi_{p^{4}}=\sin 4 x f_{p^{4}} e^{4 i t}+\sin 3 x f_{p^{43}} e^{3 i t}+\sin 2 x f_{p^{42}} e^{2 i t}+\sin x f_{p^{41}} e^{i t}+\text { c.c. }+f_{p^{4 s}} \sin 2 x,
\end{gathered}
$$


where

$$
\begin{aligned}
& F_{4}=F_{p^{4}}=0, \quad F_{43}=F_{p^{43}}=0, \\
& F_{42}=\frac{\lambda_{1}}{32 \beta}\left(\frac{\lambda \alpha^{3}}{(\alpha+i)^{2}(\alpha+2 i)}+1\right) \frac{e^{-2 \lambda_{1} \beta \bar{y}}}{2 \beta^{2}-\delta^{2}}+\frac{e^{-\lambda_{1} \beta \bar{y}}}{4 \lambda_{1} \beta\left(2 \delta^{2}-\beta^{2}\right)} \\
& +\left(\frac{\alpha^{3}(2+i) \lambda}{2(\alpha+i)^{2}(\alpha+2 i)}+\frac{\alpha^{3} \lambda_{i} \beta^{2}}{(\alpha+i)(\alpha+2 i) 4 r^{2}}\left\{\frac{\cos x}{(\alpha+2 i)\left(\beta^{2}-2 \delta^{2}\right)}+\frac{1}{2}\left(1+\lambda^{2}{ }_{1}\right)\right.\right. \\
& \left.\left.+\frac{i \beta^{2} \cos x \cos t}{2 r^{2}\left(\beta^{2}-2 \delta^{2}\right)}\right\}\right)+\frac{i}{\lambda^{2}{ }_{1} \beta^{2}-2 \delta^{2}}\left(\bar{y}+\frac{4\left(\beta^{2}-\delta^{2}\right)}{\lambda_{1} \beta\left(\beta^{2}-2 \delta^{2}\right)}\right)^{e^{-\lambda_{1} \beta \bar{y}}}, \\
& F_{p^{42}}=\frac{\alpha}{\alpha+2 i} \cdot F_{42}-\frac{\lambda^{3}}{16 \beta} \cdot \frac{\alpha^{2} e^{-2 \lambda_{1} \beta \bar{y}}}{(\alpha+i)^{2}(\alpha+2 i)} \\
& +\left(\frac{2+i}{8}\left(\frac{\alpha}{\alpha+i}\right)^{2} \frac{\lambda_{1}}{\beta(\alpha+2 i)}+\frac{\alpha^{2} i \cos x \lambda_{1} \beta}{(\alpha+i)(\alpha+2 i)^{2} 16 r^{2}\left(\beta^{2}-2 \delta^{2}\right)}\right. \\
& \left.-\frac{\alpha^{2} i \lambda^{3}{ }_{1} \beta}{(\alpha+i)^{3} 16 r^{2}(\alpha+2 i)}\right) e^{-\lambda_{1} \beta \bar{y}} \\
& F_{41}=\frac{i r^{2}}{4 \lambda_{1} \beta^{3}} e^{-\lambda_{1} \beta \bar{y}}+\frac{i r^{2}}{4 \beta^{2}} y e^{-\lambda_{1} \beta \bar{y}}+\frac{r^{2} \bar{y}^{2}}{4}-\frac{\bar{y}^{2}}{4 \lambda^{3}{ }_{1} \beta^{3}}, \quad F_{p^{41}}=\frac{\alpha}{\alpha+i} F_{41}, \\
& \frac{d F_{4 s}}{d y}=-\frac{i}{8 r}\left(\left\{\frac{1+\beta}{\beta^{3}}+\frac{\alpha \lambda_{1}}{\beta^{2}\left(\alpha^{2}+1\right)}\right\} \lambda_{1} \beta-\left\{\frac{1+\beta^{*}}{\beta^{*^{3}}}+\frac{\alpha \lambda_{1}{ }^{*}}{\beta^{* 2}\left(\alpha^{2}+1\right)}\right\} \lambda_{1}{ }^{*} \beta^{*}-\frac{\lambda_{1}(1+\beta)}{\beta^{2}} e^{-\lambda_{1} \beta \bar{y}}\right. \\
& \left.+\frac{\lambda_{1}^{*}\left(1+\beta^{*}\right)}{\beta^{*^{2}}} e^{-\lambda_{1}{ }^{*} \beta^{*} \bar{y}}-\frac{\alpha \lambda_{1}{ }^{2}}{\left(\alpha^{2}+1\right) \beta} e^{-\lambda_{1} \beta \bar{y}}+\frac{\alpha \lambda_{1} *^{2}}{\left(\alpha^{2}+1\right) \beta^{*}} e^{-\lambda_{1}{ }^{*} \beta^{*} \bar{y}}\right), \\
& \frac{d F_{p^{4 s}}}{d y}=\frac{d F_{4 s}}{d y}-\frac{i}{8 r}\left(-\frac{\beta e^{-\lambda_{1} \beta \bar{y}}}{\lambda_{1}}+\frac{\beta^{*} e^{-\lambda_{1}{ }^{*} \beta^{*} \bar{y}}}{\lambda_{1}{ }^{*}}-\frac{\alpha}{\alpha^{2}+1}\left(\lambda_{1}{ }^{4} \beta e^{-\lambda_{1} \beta \bar{y}}-\lambda_{1}{ }^{*} \beta^{*} e^{-\lambda_{1}{ }^{*} \beta^{*} \bar{y}}\right)\right) \\
& f_{4}=f_{p^{4}}=f_{43}=f_{p^{43}}=0, \quad f_{42}=\frac{1}{4\left(\beta^{2}-2 \delta^{2}\right)} e^{-2 y}, \quad f_{p^{42}}=\frac{\alpha}{\alpha+i} f_{42}, \\
& f_{41}=\frac{i r^{2}}{2 \beta^{2}} e^{-y}, \quad f_{p^{4}}=\frac{\alpha}{\alpha+i} f_{41}, \quad \frac{d f_{4 s}}{d y}=\frac{d f_{p^{4 s}}}{d y}=\frac{1}{4\left(\beta^{2}-2 \delta^{2}\right)} .
\end{aligned}
$$


210

K. KANNAN AND V. RAMAMURTHY

$$
\begin{aligned}
& \Psi=\varepsilon\left(\sin x\left(-\frac{e^{-y}}{2}\right) e^{i t}+\text { c.c. }\right)+\varepsilon^{3 / 2}\left(\sin x\left(\frac{i r \lambda_{1}}{2 \beta} e^{-\bar{y}}\right) e^{i t}+\text { c.c. }\right) \\
& +\varepsilon^{2}\left(\frac{1}{4\left(\beta^{2}-2 \delta^{2}\right)} e^{-2 y} \cdot \sin 2 x \cdot e^{2 i t}+\text { c.c. }+\frac{i r}{2 \beta^{2}} \cdot e^{-y} \cdot \sin x e^{i t}+\frac{y}{4\left(\beta^{2}-2 \delta^{2}\right)}+\text { c.c. }\right) \text {, } \\
& \Psi_{p}=\varepsilon\left(-\frac{\alpha}{\alpha+i} \sin x \frac{e^{-y}}{2} e^{i t}+\text { c.c. }\right)+\varepsilon^{3 / 2}\left(\frac{\alpha}{\alpha+i} \sin x\left(\frac{i r \lambda_{1}}{2 \beta} e^{-y}\right) e^{i t}+\text { c.c. }\right) \\
& +\varepsilon^{2}\left(\frac{\alpha}{\alpha+i} \frac{1}{4\left(\beta^{2}-2 \delta^{2}\right)} e^{-2 y} \sin 2 x e^{2 i t}+\text { c.c. }+\frac{\alpha}{\alpha+i} \frac{i r}{2 \beta^{2}} e^{-y} \sin x e^{i t}+\text { c.c. }+\frac{y}{4\left(\beta^{2}-2 \delta^{2}\right)}\right), \\
& \bar{\Psi}=\varepsilon^{1 / 2}\left(-\frac{\sin x \cos t}{r}\right)+\varepsilon\left(\sin x\left(-\frac{i \lambda_{1}}{2 \beta} e^{-\lambda_{1} \beta \bar{y}}+\frac{\bar{y}}{2}+\frac{i \lambda_{1}}{2 \beta}\right) e^{i t}+\text { c.c. }\right) \\
& +\varepsilon^{3 / 2}\left(\frac{i}{8 r\left(\beta^{2}-2 \delta^{2}\right)}\left(1-e^{-\lambda_{1} \beta \bar{y}}\right) \sin 2 x e^{2 i t}+\left(\frac{i r}{2 \beta^{2}}-\frac{i r \lambda_{1} \bar{y}}{2 \beta}-\frac{i r}{2 \beta^{2}} e^{-\lambda_{1} \beta \bar{y}}-\frac{r}{4} \bar{y}^{2}\right) \sin x e^{i t}\right. \\
& \left.+ \text { c.c. }+\frac{i}{8 r}\left\{\frac{\lambda_{1}}{\beta} e^{-\lambda_{1} \beta \bar{y}}-\frac{\lambda_{1}{ }^{*}}{\beta^{*}} e^{-\lambda^{*}{ }_{1} \beta^{*} \bar{y}}\right\}\right) \\
& +\varepsilon^{2}\left(\left(\frac{\lambda_{1}}{2 \beta}\left\{\frac{\lambda \alpha^{3}}{(\alpha+i)^{2}(\alpha+2 i)}+1\right\} \frac{e^{-2 \lambda_{1} \beta \bar{y}}}{\left(2 \beta^{2}-\delta^{2}\right)}\right.\right. \\
& +\frac{e^{-\lambda_{1} \beta \bar{y}}}{4 \lambda_{1} \beta\left(2 \delta^{2}-\beta^{2}\right)}\left\{\frac{\alpha^{3}(2+i) \lambda}{2(\alpha+i)^{2}(\alpha+2 i)}\right. \\
& +\frac{\alpha^{3} \lambda_{i} \beta^{2}}{(\alpha+i)(\alpha+2 i) 4 r^{2}}\left\{\frac{\cos x}{(\alpha+2 i)\left(\beta^{2}-2 \delta^{2}\right)}\right. \\
& \left.\left.+\frac{1}{2}\left(1+\lambda^{2}{ }_{1}\right)+\frac{i \beta^{2} \cos x \cos t}{2 r^{2}\left(\beta^{2}-2 \delta^{2}\right)}\right\}\right\} \\
& \left.+\frac{i}{\left(\lambda_{1}{ }^{2} \beta^{2}-2 \delta^{2}\right)}\left\{\bar{y}+\frac{4\left(\beta^{2}-\delta^{2}\right)}{\lambda_{1} \beta\left(\beta^{2}-2 \delta^{2}\right)}\right\} e^{-\lambda_{1} \beta \bar{y}}\right) \sin 2 x e^{2 i t} \\
& +\left\{\frac{i r^{2}}{4 \lambda_{1} \beta^{3}} e^{-\lambda_{1} \beta \bar{y}}+\frac{i r^{2}}{4 \beta^{2}} y e^{-\lambda_{1} \beta \bar{y}}+\frac{r^{2} \bar{y}^{2}}{4}-\frac{\bar{y}^{2}}{4 \lambda_{1}^{3} \beta^{3}}\right\} \sin x e^{i t}+\text { c.c. } \\
& -\frac{i}{8 r}\left(\bar{y}\left(\left\{\frac{1+\beta}{\beta^{3}}+\frac{\alpha \lambda_{1}}{\beta^{2}\left(\alpha^{2}+1\right)}\right\} \lambda_{1} \beta-\left\{\frac{1+\beta^{*}}{\beta^{* 3}}+\frac{\alpha \lambda_{1}{ }^{*}}{\beta^{*^{2}}\left(\alpha^{2}+1\right)}\right\} \lambda_{1}{ }^{*} \beta^{*}\right)\right. \\
& +\frac{1+\beta}{\beta^{3}} e^{-\lambda_{1} \beta \bar{y}}-\left(\frac{1+\beta^{*}}{\beta^{*^{3}}}\right) e^{-\lambda_{1}^{*} \beta^{*} \bar{y}}+\frac{\alpha \lambda_{1}}{\left(\alpha^{2}+1\right) \beta^{2}} e^{-\lambda_{1} \beta \bar{y}} \\
& \left.\left.-\frac{\alpha \lambda_{1}^{*}}{\left(\alpha^{2}+1\right) \beta^{*^{2}}} e^{-\lambda_{1} * \beta^{*} \bar{y}}\right) \sin 2 x\right),
\end{aligned}
$$




$$
\begin{aligned}
& \bar{\Psi}_{p}=\varepsilon^{1 / 2}\left(\frac{\alpha}{\alpha+i}\left(-\frac{1}{2 r}\right) \sin x e^{i t}+\text { c.c. }\right) \\
& +\varepsilon\left(\frac{\alpha}{\alpha+i}\left(-\frac{i \lambda_{1}}{2 \beta} e^{-\lambda_{1} \beta \bar{y}}+\frac{y}{2}+\frac{i \lambda_{1}}{2 \beta}\right) \sin x e^{i t}+\text { c.c. }\right) \\
& +\varepsilon^{3 / 2}\left(\left(\frac{i \alpha\left(1-e^{-\lambda_{1} \beta \bar{y}}\right)}{8 r\left(\beta^{2}-2 \delta^{2}\right)(\alpha+2 i)}+\frac{\alpha}{(\alpha+i)^{2}} \frac{i}{8 r}\left[\lambda_{1}{ }^{2} e^{-\lambda_{1} \beta \bar{y}}\right] \sin 2 x e^{2 i t}\right)\right. \\
& +\left(\frac{\alpha}{\alpha+i}\left\{\frac{i r}{2 \beta^{2}}-\frac{i r \lambda_{1} \bar{y}}{2 \beta}-\frac{i r}{2 \beta^{2}} e^{-\lambda_{1} \beta \bar{y}}-\frac{r}{4} \bar{y}^{2}\right\}\right) \sin x e^{i t}+\text { c.c. } \\
& \left.+\frac{i}{8 r}\left(\frac{\lambda_{1}}{\beta} e^{-\lambda_{1} \beta \bar{y}}-\frac{\lambda_{1}^{*}}{\beta^{*}} e^{-\lambda_{1}{ }^{*} \beta^{*} \bar{y}}+\frac{\alpha}{\alpha^{2}+1}\left(\frac{\lambda_{1}}{\beta} e^{-\lambda_{1} \beta \bar{y}}-\frac{\lambda_{1}^{*}}{\beta^{*}} e^{-\lambda_{1} * \beta^{*} \bar{y}}\right)\right) \sin 2 x\right) \\
& +\varepsilon^{2}\left(\left(\frac { \alpha } { \alpha + 2 i } \left\{\frac{\lambda_{1}}{32 \beta}\left(\frac{\lambda \alpha^{3}}{(\alpha+i)^{2}(\alpha+2 i)}+1\right) \frac{e^{-2 \lambda_{1} \beta \bar{y}}}{\left(2 \beta^{2}-\delta^{2}\right)}\right.\right.\right. \\
& +\frac{e^{-\lambda_{1} \beta \bar{y}}}{4 \lambda_{1} \beta\left(2 \delta^{2}-\beta^{2}\right)}\left\{\frac{\alpha^{3}(2+i) \lambda}{2(\alpha+i)^{2}(\alpha+2 i)}\right. \\
& +\frac{\alpha^{3} \lambda_{i} \beta^{2}}{(\alpha+i)(\alpha+2 i) 4 r^{2}}\left\{\frac{\cos x}{(\alpha+2 i)\left(\beta^{2}-2 \delta^{2}\right)}+\frac{1}{2}\left(1+\lambda^{2}{ }_{1}\right)\right. \\
& \left.\left.+\frac{i \beta^{2} \cos x \cos t}{2 r^{2}\left(\beta^{2}-2 \delta^{2}\right)}\right\}\right\} \\
& \left.+\frac{i}{\lambda^{2}{ }_{1} \beta^{2}-2 \delta^{2}}\left\{\bar{y}+\frac{4\left(\beta^{2}-\delta^{2}\right)}{\lambda_{1} \beta\left(\beta^{2}-2 \delta^{2}\right)}\right\} e^{-\lambda_{1} \beta \bar{y}}\right\}-\frac{\lambda_{1}{ }^{3}}{16 \beta} \frac{\alpha^{2} e^{-2 \lambda_{1} \beta \bar{y}}}{(\alpha+i)^{2}(\alpha+2 i)} \\
& +\left(\frac{2+i}{8}\left(\frac{\alpha}{\alpha+i}\right)^{2} \frac{\lambda_{1}}{\beta(\alpha+2 i)}+\frac{\alpha^{2} i \cos x \lambda_{1} \beta}{(\alpha+i)(\alpha+2 i)^{2} 16 r^{2}\left(\beta^{2}-2 \delta^{2}\right)}\right. \\
& \left.\left.-\frac{\alpha^{2} i \lambda_{1}^{3} \beta}{(\alpha+i)^{3} 16 r^{2}(\alpha+2 i)}\right) e^{-\lambda_{1} \beta \bar{y}}\right) \sin 2 x e^{2 i t} \\
& +\frac{\alpha}{\alpha+i}\left\{\frac{i r^{2}}{4 \lambda_{1} \beta^{3}} e^{-\lambda_{1} \beta \bar{y}}-\frac{i r^{2}}{4 \beta^{2}} y e^{-\lambda_{1} \beta \bar{y}}+\frac{r^{2} \bar{y}^{2}}{4}-\frac{\bar{y}^{2}}{4 \lambda^{3}{ }_{1} \beta^{3}}\right\} \sin x e^{i t} \\
& -\frac{i \sin 2 x}{8 r}\left(\bar{y}\left(\left\{\frac{1+\beta}{\beta^{3}}+\frac{\alpha \lambda_{1}}{\beta^{2}\left(\alpha^{2}+1\right)}\right\} \lambda_{1} \beta-i\left\{\frac{1+\beta^{*}}{\beta^{* 3}}+\frac{\alpha \lambda_{1}{ }^{*}}{\beta^{* 2}\left(\alpha^{2}+1\right)}\right\} \lambda_{1}{ }^{*} \beta^{*}\right)\right. \\
& +\frac{1+\beta}{\beta^{3}} e^{-\lambda_{1} \beta \bar{y}}-\frac{1+\beta^{*}}{\beta^{*}} e^{-\lambda_{1} \beta^{*} \bar{y}}+\frac{\alpha \lambda_{1}}{\left(\alpha^{2}+1\right) \beta^{2}} e^{-\lambda_{1} \beta \bar{y}}-\frac{\alpha \lambda_{1}{ }^{*}}{\left(\alpha^{2}+1\right) \beta^{*^{2}}} e^{-\lambda_{1}{ }^{*} \beta^{*} \bar{y}} \\
& \left.+\frac{e^{-\lambda_{1} \beta \bar{y}}}{\lambda_{1}{ }^{2}}-\frac{e^{-\lambda_{1} * \beta^{*} \bar{y}}}{\lambda_{1}{ }^{2}}-\frac{\alpha}{\alpha^{2}+1}\left(-\lambda_{1}{ }^{3} e^{-\lambda_{1} \beta \bar{y}}+\lambda_{1}{ }^{3 *} e^{-\lambda_{1}{ }^{*} \beta^{*}}\right)\right) .
\end{aligned}
$$


From the above expressions of $\Psi(x, y, t)$ and $\Psi_{p}(x, y, t)$ representing the stream functions of the fluid and, dust, respectively, the following expressions for the velocity components for inner and outer flow have been obtained

$$
\begin{aligned}
& U_{i}=\left(\frac{\sin x}{2}\left(i \lambda_{1}^{2} e^{-\lambda_{1} \beta \bar{y}}+1\right) e^{i t}+\text { c.c. }\right) \varepsilon \\
& +\varepsilon^{3 / 2}\left(\frac{i \lambda_{1} \beta}{8 r} e^{-\lambda_{1} \beta \bar{y}} \sin 2 x \frac{e^{2 i t}}{\beta^{2}-2 \delta^{2}}+\frac{\sin x}{2}\left(\frac{i r \lambda_{1}}{\beta} e^{-\lambda_{1} \beta \bar{y}}-r \bar{y}-\frac{i r \lambda_{1}}{\beta}\right) e^{i t}\right. \\
& \left.+ \text { c.c. }+\left(\frac{i}{8 r} \sin 2 x\left(\lambda_{1}{ }^{*} e^{-\lambda_{1} *^{*} \bar{y}}-\lambda_{1}{ }^{2} e^{-\lambda_{1} \beta \bar{y}}\right)\right)\right) \\
& +\varepsilon^{2}\left[\left[\frac{-\lambda^{2}{ }_{1} e^{-2 \lambda_{1} \beta \bar{y}}}{16\left(2 \beta^{2}-\delta^{2}\right)}\left(\frac{\lambda \alpha^{3}}{(\alpha+i)^{2}(\alpha+2 i)}+1\right)\right.\right. \\
& -\frac{e^{-\lambda_{1} \beta \bar{y}}}{4\left(2 \delta^{2}-\beta^{2}\right)}\left\{\frac{\alpha^{3}(2+i) \lambda}{2(\alpha+i)^{2}(\alpha+2 i)}\right. \\
& +\frac{\alpha^{3} \lambda_{i} \beta^{2}}{(\alpha+i)(\alpha+2 i) 4 r^{2}}\left(\frac{\cos x}{(\alpha+2 i)\left(\beta^{2}-2 \delta^{2}\right)}\right. \\
& \left.\left.+\frac{1}{2}\left(1+\lambda_{1}^{2}\right)+\frac{i \beta^{2} \cos x \cos t}{2 r^{2}\left(\beta^{2}-2 \delta^{2}\right)}\right)\right\} \\
& \left.+\frac{i e^{-\lambda_{1} \beta y}}{\left(\lambda^{2}{ }_{1} \beta^{2}-2 \delta^{2}\right)}\left\{1-\left(\lambda_{1} \beta \bar{y}+\frac{4\left(\beta^{2}-\delta^{2}\right)}{\left(\beta^{2}-2 \delta^{2}\right)}\right)\right\}\right] \sin 2 x e^{2 i t} \\
& +\left\{-\frac{i r^{2}}{4 \beta} e^{-\lambda_{1} \beta \bar{y}}+\frac{i r^{2}}{4 \beta^{2}}\left(1-\lambda_{1} \beta y\right) e^{-\lambda_{1} \beta \bar{y}}+\frac{r^{2} \bar{y}}{2}-\frac{\bar{y}}{2 \lambda^{3}{ }_{1} \beta^{3}}\right\} \sin x e^{i t} \\
& -\frac{i}{8 r}\left\{\left(\frac{1+\beta}{\beta^{3}}+\frac{\alpha \lambda_{1}}{\beta^{2}\left(\alpha^{2}+1\right)}\right) \lambda_{1} \beta-\left(\frac{1+\beta^{*}}{\beta^{*^{3}}}+\frac{\alpha \lambda_{1}{ }^{*}}{\beta^{*^{2}}\left(\alpha^{2}+1\right)}\right) \lambda_{1}{ }^{*} \beta^{*}\right. \\
& -\frac{\lambda_{1}(1+\beta)}{\beta^{2}} e^{-\lambda_{1} \beta \bar{y}}+\frac{\lambda_{1}{ }^{*}\left(1+\beta^{*}\right)}{\beta^{*^{2}}} e^{-\lambda^{*}{ }_{1} \beta^{*} \bar{y}}-\frac{\alpha \lambda^{2}{ }_{1}}{\beta\left(\alpha^{2}+1\right)} e^{-\lambda_{1} \beta \bar{y}} \\
& \left.\left.-\frac{\alpha \lambda_{1}{ }^{2}}{\beta^{*}\left(\alpha^{2}+1\right)} e^{-\lambda_{1} \beta^{*} \bar{y}}\right\} \sin 2 x\right] \text {, } \\
& U_{p^{i}}=\varepsilon\left(\frac{\alpha}{\alpha+i} \frac{\sin x}{2}\left(i \lambda_{1}{ }^{2} e^{-\lambda_{1} \beta \bar{y}}+1\right) e^{i t}+\text { c.c. }\right) \\
& +\varepsilon^{3 / 2}\left[\left(\frac{\alpha}{\alpha+2 i}\left(\frac{i \lambda_{1} \beta}{8 r} \frac{e^{-\lambda_{1} \beta \bar{y}}}{\beta^{2}-2 \delta^{2}}\right)-\frac{i}{8 r} \frac{\alpha \beta \lambda_{1}^{3} e^{-\lambda_{1} \beta \bar{y}}}{(\alpha+i)^{2}}\right) \sin 2 x e^{2 i t}\right.
\end{aligned}
$$




$$
\begin{aligned}
& +\frac{\alpha \sin x}{2(\alpha+i)}\left(\frac{i r \lambda_{1}}{\beta} e^{-\lambda_{1} \beta \bar{y}}-r y-i r \lambda_{1}\right) e^{i t} \\
& + \text { c.c. }+\frac{i}{8 r} \sin 2 x\left\{\left(\lambda_{1} *^{2} e^{-\lambda_{1} *^{*} \bar{y}}-\lambda_{1}{ }^{2} e^{-\lambda_{1} \beta \bar{y}}\right)\right. \\
& \left.\left.+\frac{\alpha}{\alpha^{2}+1}\left(\lambda_{1} *^{3} e^{-\lambda_{1} * \beta^{*} \bar{y}}-\lambda_{1}^{3} e^{-\lambda_{1} \beta \bar{y}}\right)\right\}\right] \\
& +\varepsilon^{2}\left[( \frac { \alpha } { \alpha + 2 i } ) \left[-\frac{\lambda_{1}{ }^{2} e^{-2 \lambda_{1} \beta \bar{y}}}{16\left(2 \beta^{2}-\delta^{2}\right)}\left(\frac{\lambda \alpha^{3}}{(\alpha+i)^{2}(\alpha+2 i)}+1\right)\right.\right. \\
& -\frac{e^{-\lambda_{1} \beta \bar{y}}}{4\left(2 \delta^{2}-\beta^{2}\right)}\left\{\frac{\alpha^{3}(2+i) \lambda}{2(\alpha+i)^{2}(\alpha+2 i)}\right. \\
& +\frac{\alpha^{3} \lambda_{i} \beta^{2}}{(\alpha+i)(\alpha+2 i) 4 r^{2}}\left(\frac{\cos x}{(\alpha+2 i)\left(\beta^{2}-2 \delta^{2}\right)}+\frac{1}{2}\left(1+\lambda_{1}{ }^{2}\right)\right. \\
& \left.\left.+\frac{i \beta^{2} \cos x \cos t}{2 r^{2}\left(\beta^{2}-2 \delta^{2}\right)}\right)\right\} \\
& \left.+\frac{i e^{-\lambda_{1} \beta \bar{y}}}{\left(\lambda^{2}{ }_{1} \beta^{2}-2 \delta^{2}\right)}\left\{1-\left\{\lambda_{1} \beta \bar{y}+\frac{4\left(\beta^{2}-\delta^{2}\right)}{\left(\beta^{2}-2 \delta^{2}\right)}\right\}\right\}\right] \sin 2 x e^{2 i t}+\frac{\lambda_{1}{ }^{4} \alpha e^{-2 \lambda_{1} \beta \bar{y}}}{8(\alpha+i)^{2}} \\
& -\lambda_{1} \beta e^{-\lambda_{1} \beta \bar{y}}\left(\frac{(2+i) \lambda_{1} \alpha}{8 \beta(\alpha+i)^{2}}\right. \\
& \left.\left.+\frac{\alpha i \lambda_{1} \beta \cos x}{(\alpha+i)(\alpha+2 i) 16 r^{2}\left(\beta^{2}-2 \delta^{2}\right)}-\frac{\alpha i \lambda_{1}^{3} \beta}{(\alpha+i)^{3} 16 r^{2}}\right)\right] \sin 2 x e^{2 i t} \\
& +\frac{\alpha}{\alpha+i}\left(-\frac{i r^{2}}{4 \beta} e^{-\lambda_{1} \beta \bar{y}}+\frac{i r^{2}}{4 \beta^{2}}\left(1-\lambda_{1} \beta \bar{y}\right) e^{-\lambda_{1} \beta \bar{y}}+\frac{r^{2} \bar{y}}{2}-\frac{\bar{y}}{2 \lambda_{1}^{3} \beta^{3}}\right) \sin x e^{i t} \\
& -\frac{i}{8 r}\left[\left\{\left[\frac{1+\beta}{\beta^{3}}+\frac{\alpha \lambda_{1}}{\beta^{2}\left(\alpha^{2}+1\right)}\right] \lambda_{1} \beta-\left(\frac{1+\beta^{*}}{\beta^{*}}+\frac{\alpha \lambda^{*}{ }_{1}}{\beta^{* 2}\left(\alpha^{2}+1\right)}\right) \lambda_{1}{ }^{*} \beta^{*}\right.\right. \\
& -\frac{\lambda_{1}(1+\beta)}{\beta^{2}} e^{-\lambda_{1} \beta \bar{y}}+\frac{\lambda_{1}^{*}\left(1+\beta^{*}\right)}{\beta^{*^{2}}} e^{-\lambda_{1}^{*} \beta^{*} \bar{y}} \\
& \left.-\frac{\alpha \lambda^{2}{ }_{1}}{\beta\left(\alpha^{2}+1\right)} e^{-\lambda_{1} \beta \bar{y}}+\frac{\alpha \lambda_{1} *^{2}}{\beta^{*}\left(\alpha^{2}+1\right)} e^{-\lambda_{1} \beta^{*} \bar{y}}\right\} \\
& \left.-\frac{\beta e^{-\lambda_{1} \beta \bar{y}}}{\lambda_{1}}+\frac{\beta^{*} e^{-\lambda_{1}{ }^{*} \beta^{*} \bar{y}}}{\lambda_{1}{ }^{*}}-\frac{\alpha}{\alpha^{2}+1}\left(\lambda_{1}{ }^{4} \beta e^{-\lambda_{1} \beta \bar{y}}-\lambda_{1}{ }^{4} \beta^{*} e^{-\lambda_{1}{ }^{*} \beta^{*} \bar{y}}\right) \sin 2 x\right] \text {, }
\end{aligned}
$$


214

K. KANNAN AND V. RAMAMURTHY

$$
\begin{aligned}
& V_{i}=\varepsilon^{1 / 2}\left(\frac{\cos x \cos t}{r}\right)+\varepsilon\left(\frac{\cos x}{2}\left(\frac{i \lambda_{1}}{\beta} e^{-\lambda_{1} \beta \bar{y}}-y-\frac{i \lambda_{1}}{\beta}\right) e^{i t}+\text { c.c. }\right) \\
& +\varepsilon^{3 / 2}\left(\frac{i}{4 r}\left(\frac{\cos 2 x\left(e^{-\lambda_{1} \beta \bar{y}}-1\right)}{\beta^{2}-2 \delta^{2}}\right) e^{2 i t}+\cos x\left(\frac{i r}{2 \beta^{2}} e^{-\lambda_{1} \beta \bar{y}}+\frac{r \bar{y}^{2}}{4}+\frac{i r \lambda_{1}}{2 \beta}-\frac{i r}{2 \beta^{2}}\right) e^{i t}\right. \\
& \left.+ \text { c.c. }+\frac{i}{4 r}\left(\frac{\lambda_{1}^{*}}{\beta^{*}} e^{-\lambda_{1}{ }^{*} \beta^{*} \bar{y}}-\frac{\lambda_{1}}{\beta} e^{-\lambda_{1} \beta \bar{y}}\right) \cos 2 x\right) \\
& +\varepsilon^{2}\left[e ^ { 2 i t } \left(-2 \cos 2 x\left[\frac{\lambda_{1}}{32 \beta}\left\{\frac{\lambda \alpha^{3}}{(\alpha+i)^{2}(\alpha+2 i)}+1\right\} \frac{e^{-2 \lambda_{1} \beta \bar{y}}}{\left(2 \beta^{2}-\delta^{2}\right)}\right.\right.\right. \\
& +\frac{e^{-\lambda_{1} \beta \bar{y}}}{4 \lambda_{1} \beta\left(2 \delta^{2}-\beta^{2}\right)}\left\{\frac{\alpha^{3}(2+i) \lambda}{2(\alpha+i)^{2}(\alpha+2 i)}+\frac{\alpha^{3} \lambda i \beta^{2}}{(\alpha+i)(\alpha+2 i) 4 r^{2}}\right. \\
& \times\left\{\frac{\cos x}{(\alpha+2 i)\left(\beta^{2}-2 \delta^{2}\right)}+\frac{1}{2}\left(1+\lambda_{1}^{2}\right)\right. \\
& \left.\left.+\frac{i \beta^{2} \cos x \cos t}{2 r^{2}\left(\beta^{2}-2 \delta^{2}\right)}\right\}\right\} \\
& \left.+\frac{i}{\left(\lambda_{1}^{2} \beta^{2}-2 \delta^{2}\right)}\left\{\bar{y}+\frac{4\left(\beta^{2}-\delta^{2}\right)}{\lambda_{1} \beta\left(\beta^{2}-2 \delta^{2}\right)}\right\} e^{-\lambda_{1} \beta \bar{y}}\right] \\
& +\sin 2 x\left(\frac { e ^ { - \lambda _ { 1 } \beta \overline { y } } \alpha ^ { 3 } \lambda i \beta } { 1 6 \lambda _ { 1 } ( 2 \delta ^ { 2 } - \beta ^ { 2 } ) ( \alpha + i ) ( \alpha + 2 i ) r ^ { 2 } } \left\{\frac{\sin x}{(\alpha+2 i)\left(\beta^{2}-2 \delta^{2}\right)}\right.\right. \\
& \left.\left.\left.+\frac{i \beta^{2} \sin x \cos t}{2 r^{2}\left(\beta^{2}-2 \delta^{2}\right)}\right\}\right)\right) \\
& -\cos x\left\{e^{i t}\left\{\frac{i r^{2}}{4 \lambda_{1} \beta^{3}} e^{-\lambda_{1} \beta \bar{y}}+\frac{i r^{2}}{4 \beta^{2}} \bar{y} e^{-\lambda_{1} \beta \bar{y}}+\frac{r^{2} \bar{y}^{2}}{4}-\frac{\bar{y}^{2}}{4 \lambda_{1}{ }^{3} \beta^{3}}\right\}+\text { c.c. }\right\} \\
& +\frac{i}{4 r} \cos 2 x\left(\left(\bar{y}\left\{\frac{1+\beta}{\beta^{3}}+\frac{\alpha \lambda_{1}}{\beta^{2}\left(\alpha^{2}+1\right)}\right\} \lambda_{1} \beta-\left\{\frac{1+\beta^{*}}{\beta^{3 *}}+\frac{\alpha \lambda_{1}{ }^{*}}{\beta^{* 2}\left(\alpha^{2}+1\right)}\right\} \lambda_{1}{ }^{*} \beta^{*}\right)\right) \\
& \left.+\frac{1+\beta}{\beta^{3}} e^{-\lambda_{1} \beta \bar{y}}-\frac{1+\beta^{*}}{\beta^{*^{3}}} e^{-\lambda_{1}{ }^{*} \beta^{*} \bar{y}}+\frac{\alpha \lambda_{1}}{\left(\alpha^{2}+1\right) \beta^{2}} e^{-\lambda_{1} \beta \bar{y}}-\frac{\alpha \lambda_{1}^{*}}{\left(\alpha^{2}+1\right) \beta^{*^{2}}} e^{-\lambda_{1}{ }^{*} \beta^{*} \bar{y}}\right],
\end{aligned}
$$

$$
\begin{aligned}
V_{p^{i}}= & \varepsilon^{1 / 2}\left(\frac{\alpha}{\alpha+i} \frac{1}{2 r} \cos x e^{i t}+\text { c.c. }\right)+\varepsilon\left(\frac{\alpha \cos x}{2(\alpha+i)}\left\{\frac{i \lambda_{1}}{\beta} e^{-\lambda_{1} \beta \bar{y}}-\bar{y}-\frac{i \lambda_{1}}{\beta}\right\} e^{i t}+\text { c.c. }\right) \\
& +\varepsilon^{3 / 2}\left(\left\{\frac{\alpha}{\alpha+2 i}\left\{\frac{i}{4 r} \frac{\cos 2 x}{\beta^{2}-2 \delta^{2}}\left(e^{-\lambda_{1} \beta \bar{y}}-1\right)\right\}-\frac{\alpha}{(\alpha+i)^{2}} \frac{i}{4 r} \cos 2 x \lambda_{1}^{2} e^{-\lambda_{1} \beta \bar{y}}\right\} e^{2 i t}\right.
\end{aligned}
$$




$$
\begin{aligned}
& +\frac{\alpha}{\alpha+i}\left(\cos x\left(\frac{i r}{2 \beta^{2}} e^{-\lambda_{1} \beta \bar{y}}+\frac{r \bar{y}^{2}}{4}+\frac{i r \lambda_{1}}{2 \beta}-\frac{i r}{2 \beta^{2}}\right)\right) e^{i t}+\text { c.c. } \\
& \left.+\frac{i}{4 r}\left\{\frac{\alpha^{2}+\alpha+1}{\alpha^{2}+1} \cos 2 x\left\{\frac{\lambda_{1}^{*}\left(1+\lambda_{1}^{*}\right)}{\beta^{*}} e^{-\lambda_{1} \beta^{*} \bar{y}}-\frac{\lambda_{1}\left(1+\lambda_{1}\right)}{\beta} e^{-\lambda_{1} \beta \bar{y}}\right\}\right\}\right) \\
& +\varepsilon^{2}\left[\left\{-2 \cos 2 x\left[\frac { \alpha } { \alpha + 2 i } \left[\frac{\lambda_{1}}{32 \beta}\left\{\frac{\lambda \alpha^{3}}{(\alpha+i)^{2}(\alpha+2 i)}+1\right\}+\frac{e^{-2 \lambda_{1} \beta y}}{\left(2 \beta^{2}-\delta^{2}\right)}\right.\right.\right.\right. \\
& +\frac{e^{-\lambda_{1} \beta y}}{4 \lambda_{1} \beta\left(2 \delta^{2}-\beta^{2}\right)}\left\{\frac{\alpha^{3}(2+i) \lambda}{2(\alpha+i)^{2}(\alpha+2 i)}+\frac{\alpha^{3} \lambda_{i} \beta^{2}}{(\alpha+i)(\alpha+2 i) 4 r^{2}}\right. \\
& \times\left\{\frac{\cos x}{(\alpha+2 i)\left(\beta^{2}-2 \delta^{2}\right)}+\frac{1}{2}\left(1+\lambda^{2}{ }_{1}\right)\right. \\
& \left.\left.+\frac{i \beta^{2} \cos x \cos t}{2 r^{2}\left(\beta^{2}-2 \delta^{2}\right)}\right\}\right\} \\
& \left.+\frac{i}{\lambda^{2}{ }_{1} \beta^{2}-2 \delta^{2}}\left\{y+\frac{4\left(\beta^{2}-\delta^{2}\right)}{\lambda_{1} \beta\left(\beta^{2}-2 \delta^{2}\right)}\right\} e^{-\lambda_{1} \beta y}\right] \\
& -\frac{\lambda^{3}{ }_{1}}{16 \beta} \frac{\alpha^{2} e^{-2 \lambda_{1} \beta y}}{(\alpha+i)^{2}(\alpha+2 i)} \\
& +\left\{\frac{2+i}{8}\left(\frac{\alpha}{\alpha+i}\right)^{2} \frac{\lambda_{1}}{\beta(\alpha+2 i)}+\frac{\alpha^{2} i \cos x \lambda_{1} \beta}{(\alpha+i)(\alpha+2 i)^{2} 16 r^{2}\left(\beta^{2}-2 \delta^{2}\right)}\right. \\
& \left.\left.-\frac{\alpha^{2} i \lambda_{1}^{3} \beta}{(\alpha+i)^{3} 16 r^{2}(\alpha+2 i)}\right\} e^{-\lambda_{1} \beta y}\right] \\
& +\sin 2 x\left[\frac{e^{-\lambda_{1} \beta y} \alpha^{3} \lambda i \beta}{16 \lambda_{1}\left(2 \delta^{2}-\beta^{2}\right)(\alpha+i)(\alpha+2 i) r^{2}}\left\{\frac{\sin x}{(\alpha+2 i)\left(\beta^{2}-2 \delta^{2}\right)}+\frac{i \beta^{2} \sin x \cos t}{2 r^{2}\left(\beta^{2}-2 \delta^{2}\right)}\right\}\right. \\
& \left.\left.-\frac{\lambda_{1}^{4} \alpha^{4} i \sin x e^{-2 \lambda_{1} \beta y}}{(\alpha+i)^{3}(\alpha+2 i) 256 r^{2}\left(\beta^{2}-2 \delta^{2}\right)}+\text { c.c. }\right]\right\} e^{2 i t} \\
& -\frac{\alpha}{\alpha+i} \cos x\left\{e^{i t}\left\{\frac{i r^{2}}{4 \lambda_{1} \beta^{3}} e^{-\lambda_{1} \beta y}+\frac{i r^{2}}{4 \beta^{2}} y e^{-\lambda_{1} \beta y}+\frac{r^{2} y^{2}}{4}-\frac{y^{2}}{4 \lambda_{1}^{3} \beta^{3}}\right\}+\text { c.c. }\right\} \\
& +\frac{i \cos 2 x}{4 r}\left\{\left(y\left\{\frac{1+\beta}{\beta^{3}}+\frac{\alpha \lambda_{1}}{\beta^{2}\left(\alpha^{2}+1\right)}\right\} \lambda_{1} \beta-i\left(\frac{1+\beta^{*}}{\beta^{* 3}}+\frac{\alpha \lambda_{1}{ }^{*}}{\beta^{* 2}\left(\alpha^{2}+1\right)}\right) \lambda^{*}{ }_{1} \beta^{*}\right)\right. \\
& +\frac{1+\beta}{\beta^{3}} e^{-\lambda_{1} \beta y}-\frac{1+\beta^{*}}{\beta^{*}} e^{-\lambda^{*}{ }_{1} \beta^{*} y}+\frac{\alpha \lambda_{1}}{\left(\alpha^{2}+1\right) \beta^{2}} e^{-\lambda_{1} \beta y} \\
& -\frac{\alpha \lambda_{1}^{*}}{\left(\alpha^{2}+1\right) \beta^{*^{2}}} e^{-\lambda_{1} \beta^{*} y}+\frac{e^{-\lambda_{1} \beta y}}{\lambda^{2}{ }_{1}}-\frac{e^{-\lambda_{1} *^{*} y}}{\lambda_{1}{ }^{2}} \\
& \left.\left.-\frac{\alpha}{\alpha^{2}+1}\left(-\lambda_{1}{ }^{3} e^{-\lambda_{1} \beta y}+\lambda_{1} *^{3} e^{-\lambda_{1} *^{*} y}\right)\right\}\right] \text {, }
\end{aligned}
$$




$$
\begin{aligned}
& U_{i s}=\frac{i}{8 r} \sin 2 x\left(-\lambda_{1}{ }^{2} e^{-\lambda_{1} \beta \bar{y}}+\lambda_{1}{ }^{2} e^{-\lambda_{1}{ }^{*} \beta^{*} \bar{y}}\right) \varepsilon^{3 / 2} \\
& +\varepsilon^{2}\left[-\frac{i}{8 r} \sin 2 x\left\{\left(\frac{1+\beta}{\beta^{3}}+\frac{\alpha \lambda_{1}}{\beta^{2}\left(\alpha^{2}+1\right)}\right) \lambda_{1} \beta-\left(\frac{1+\beta^{*}}{\beta^{*}}+\frac{\alpha \lambda_{1}{ }^{*}}{\beta^{* 2}\left(\alpha^{2}+1\right)}\right) \lambda_{1}{ }^{*} \beta^{*}\right.\right. \\
& -\frac{\lambda_{1}(1+\beta)}{\beta^{2}} e^{-\lambda_{1} \beta \bar{y}}+\frac{\lambda_{1}^{*}\left(1+\beta^{*}\right)}{\beta^{*^{2}}} e^{-\lambda_{1}^{*} \beta^{*} \bar{y}} \\
& \left.\left.-\frac{\alpha \lambda_{1}{ }^{2}}{\beta\left(\alpha^{2}+1\right)} e^{-\lambda_{1} \beta \bar{y}}+\frac{-\alpha \lambda_{1}{ }^{2}}{\beta^{*}\left(\alpha^{2}+1\right)} e^{-\lambda_{1} \beta^{*} \bar{y}}\right\}\right] \text {, } \\
& U_{p^{i s}}=\frac{i \sin 2 x}{8 r}\left(\left(\lambda_{1}{ }^{*^{2}} e^{-\lambda_{1}{ }^{*} \beta^{*} y}-\lambda_{1}{ }^{2} e^{-\lambda_{1} \beta y}\right)+\frac{\alpha}{\alpha^{2}+1}\left(\lambda_{1}{ }^{3} e^{-\lambda_{1} \beta^{*} y}-\lambda_{1}{ }^{3} e^{-\lambda_{1} \beta y}\right)\right) \varepsilon^{3 / 2} \\
& -\frac{i}{8 r} \sin 2 x \varepsilon^{2}\left[\left(\left\{\frac{1+\beta}{\beta^{3}}+\frac{\alpha \lambda_{1}}{\beta^{2}\left(\alpha^{2}+1\right)}\right\} \lambda_{1} \beta-\left(\frac{1+\beta^{*}}{\beta^{*^{3}}}+\frac{\alpha \lambda_{1}{ }^{*}}{\beta^{*^{2}}\left(\alpha^{2}+1\right)}\right) \lambda_{1}{ }^{*} \beta^{*}\right.\right. \\
& -\frac{\lambda_{1}(1+\beta)}{\beta^{2}} e^{-\lambda_{1} \beta y}+\frac{\lambda_{1}^{*}\left(1+\beta^{*}\right)}{\beta^{*^{2}}} e^{-\lambda_{1}^{*} \beta^{*} y}-\frac{\alpha \lambda_{1}{ }^{2}}{\beta\left(\alpha^{2}+1\right)} e^{-\lambda_{1} \beta y} \\
& \left.+\frac{\alpha \lambda_{1}{ }^{2}}{\beta^{*}\left(\alpha^{2}+1\right)} e^{-\lambda_{1}{ }^{*} \beta^{*} y}-\frac{\beta e^{-\lambda_{1} \beta y}}{\lambda_{1}}+\frac{\beta^{*} e^{-\lambda_{1}{ }^{*} \beta^{*} y}}{\lambda_{1}{ }^{*}}\right) \\
& \left.-\frac{\alpha}{\alpha^{2}+1}\left(\lambda_{1}^{4} \beta e^{-\lambda_{1} \beta y}-\lambda_{1}{ }^{4} \beta^{*} e^{-\lambda_{1} * \beta^{*} y}\right)\right] \text {, } \\
& V_{i s}=\frac{i}{4 r} \cos 2 x\left(\frac{\lambda_{1}^{*}}{\beta^{*}} e^{-\lambda_{1} * \beta^{*} \bar{y}}-\frac{\lambda_{1}}{\beta} e^{-\lambda_{1} \beta \bar{y}}\right) \varepsilon^{3 / 2} \\
& +\varepsilon^{2} \frac{i \cos 2 x}{4 r}\left(\bar{y}\left(\left[\frac{1+\beta}{\beta^{3}}+\frac{\alpha \lambda_{1}}{\beta^{2}\left(\alpha^{2}+1\right)}\right] \lambda_{1} \beta+\left\{-\frac{1+\beta^{*}}{\beta^{3 *}}+\frac{\alpha \lambda_{1}{ }^{*}}{\beta^{2 *}\left(\alpha^{2}+1\right)}\right\} \lambda_{1}{ }^{*} \beta^{*}\right)\right. \\
& +\frac{1+\beta}{\beta^{3}} e^{-\lambda_{1} \beta \bar{y}}-\frac{1+\beta^{*}}{\beta^{*^{3}}} e^{-\lambda_{1} * \beta^{*} \bar{y}}+\frac{\alpha \lambda_{1}}{\left(\alpha^{2}+1\right) \beta^{2}} e^{-\lambda_{1} \beta \bar{y}} \\
& -\frac{\alpha \lambda_{1}{ }^{*}}{\left(\alpha^{2}+1\right) \beta^{*^{2}}} e^{-\lambda_{1}{ }^{*} \beta^{*} \bar{y}}+\frac{1+\beta}{\beta^{3}} e^{-\lambda_{1} \beta \bar{y}}-\frac{1+\beta^{*}}{\beta^{3 *}} e^{-\lambda_{1}{ }^{*} \beta^{*} \bar{y}} \\
& \left.+\frac{\alpha \lambda_{1}}{\left(\alpha^{2}+1\right) \beta^{2}} e^{-\lambda_{1} \beta \bar{y}}-\frac{\alpha \lambda_{1}^{*}}{\left(\alpha^{2}+1\right) \beta^{* 2}} e^{-\lambda_{1} \beta^{*} \bar{y}}\right),
\end{aligned}
$$

$$
\begin{aligned}
V_{p^{i s}}= & \frac{i \cos 2 x}{4 r}\left(\left\{\frac{\lambda_{1}{ }^{*}}{\beta^{*}} e^{-\lambda_{1}{ }^{*} \beta^{*} y}-\frac{\lambda_{1}}{\beta} e^{-\lambda_{1} \beta y}\right\}+\frac{\alpha}{\alpha^{2}+1}\left\{\frac{\lambda_{1} *^{2}}{\beta^{*}} e^{-\lambda_{1}{ }^{*} \beta^{*} y}-\frac{\lambda_{1}{ }^{2}}{\beta} e^{-\lambda_{1} \beta y}\right\}\right) \varepsilon^{3 / 2} \\
& +\varepsilon^{2}\left[\frac{i \cos 2 x}{4 r} y\left[\left\{\frac{1+\beta}{\beta^{3}}+\frac{\alpha \lambda_{1}}{\beta^{2}\left(\alpha^{2}+1\right)}\right\} \lambda_{1} \beta-i\left\{\frac{1+\beta^{*}}{\beta^{*}}+\frac{\alpha \lambda_{1}{ }^{*}}{\beta^{*}\left(\alpha^{2}+1\right)}\right\} \lambda_{1}{ }^{*} \beta^{*}\right]\right.
\end{aligned}
$$




$$
\begin{aligned}
& +\frac{1+\beta}{\beta^{3}} e^{-\lambda_{1} \beta y}-\frac{1+\beta^{*}}{\beta^{*}} e^{-\lambda_{1} * \beta^{*} y}+\frac{\alpha \lambda_{1}}{\left(\alpha^{2}+1\right) \beta^{2}} e^{-\lambda_{1} \beta y} \\
& -\frac{\alpha \lambda_{1}{ }^{*}}{\left(\alpha^{2}+1\right) \beta^{*^{2}}} e^{-\lambda_{1}{ }^{*} \beta^{*} y}+\frac{e^{-\lambda_{1} \beta y}}{\lambda_{1}^{2}}-\frac{e^{-\lambda_{1} \beta^{*} y}}{\lambda_{1}{ }^{2}} \\
& \left.-\frac{\alpha}{\alpha^{2}+1}\left(-\lambda^{3}{ }_{1} e^{-\lambda_{1} \beta y}+\lambda_{1} *^{3} e^{-\lambda_{1} * \beta^{*} y}\right)\right] \text {, } \\
& U_{o}=\varepsilon\left(\frac{\sin x}{2} e^{-y} e^{i t}+\text { c.c. }\right)+\varepsilon^{3 / 2}\left(-\frac{i r \lambda_{1}}{2 \beta} e^{-y} e^{i t} \sin x+\text { c.c. }\right) \\
& +\varepsilon^{2}\left(-\frac{e^{-2 y}}{2\left(\beta^{2}-2 \delta^{2}\right)} \sin 2 x e^{2 i t}+\text { c.c. }-\frac{i r}{2 \beta^{2}} e^{-y} \sin x e^{i t}+\text { c.c. }+\frac{1}{4\left(\beta^{2}-2 \delta^{2}\right)}\right), \\
& U_{p^{o}}=\varepsilon\left(\frac{\sin x}{2} \frac{\alpha}{\alpha+i} e^{-y} e^{i t}+\text { c.c. }\right)+\varepsilon^{3 / 2}\left(\frac{i r \lambda_{1}}{2 \beta} \sin x \frac{\alpha}{\alpha+i} e^{-y} e^{i t}+\text { c.c. }\right) \\
& +\varepsilon^{2}\left(-\frac{\alpha}{\alpha+i} \frac{1}{2\left(\beta^{2}-2 \delta^{2}\right)} e^{-2 y} \sin 2 x e^{2 i t}+\right.\text { c.c. } \\
& \left.-\frac{\alpha}{\alpha+i} \frac{i r}{2 \beta^{2}} e^{-y} \sin x e^{i t}+\text { c.c. }+\frac{1}{4\left(\beta^{2}-2 \delta^{2}\right)}\right), \\
& V_{o}=\varepsilon\left(\frac{\cos x}{2} e^{-y} e^{i t}+\text { c.c. }\right)+\varepsilon^{3 / 2}\left(-\frac{i r \lambda_{1}}{2 \beta} e^{-y} e^{i t} \cos x+\text { c.c. }\right) \\
& -\varepsilon^{2}\left(\frac{1}{4\left(\beta^{2}-2 \delta^{2}\right)} e^{-2 y} \cos 2 x e^{2 i t}+\text { c.c. }+\frac{i r}{2 \beta^{2}} e^{-y} \cos x e^{i t}+\text { c.c. }\right), \\
& V_{p^{o}}=\varepsilon\left(\frac{\cos x}{2} \frac{\alpha}{\alpha+i} e^{-y} e^{i t}+\text { c.c. }\right)+\varepsilon^{3 / 2}\left(\frac{-i r \lambda_{1}}{2 \beta} \cos x \frac{\alpha}{\alpha+i} e^{-y} e^{i t}+\text { c.c. }\right) \\
& -\varepsilon^{2}\left(\frac{\alpha}{\alpha+i} \frac{1}{4\left(\beta^{2}-2 \delta^{2}\right)} e^{-2 y} \cos 2 x e^{2 i t}+\text { c.c. }+\frac{\alpha}{\alpha+i} \frac{i r}{2 \beta^{2}} e^{-y} \cos x e^{i t}+\text { c.c. }\right) \text {, }
\end{aligned}
$$

where

$\partial \bar{\Psi} / \partial \bar{y}=U_{i}$ represents the inner axial velocity of the component of the fluid. $\partial \bar{\Psi} / \partial \bar{y}=U_{p^{i}}$ represents the inner axial velocity component of dust particles. $-\partial \bar{\Psi} / \partial x=V_{i}$ represents the inner transverse velocity component of the fluid. $-\partial \Psi_{p} / \partial x=V_{p^{i}}$ represents the inner transverse velocity component of dust. $U_{i s}$ represents the inner steady axial velocity component of the fluid. $U_{p^{i s}}$ represents the inner steady axial velocity component of dust. $V_{i s}$ represents the inner steady transverse velocity component of the fluid. $V_{p i s}$ represents the inner steady transverse velocity component of the dust. $\partial \Psi / \partial y=U_{o}$ represents the outer axial velocity component of the fluid.

$\partial \Psi_{p} / \partial y=U_{p^{o}}$ represents the outer axial velocity of the component of the dust. $-\partial \Psi / \partial x=V_{o}$ represents the outer transverse velocity component of the fluid. $-\partial \Psi_{p} / \partial x=V_{p^{o}}$ represents the outer transverse velocity component of the dust. 


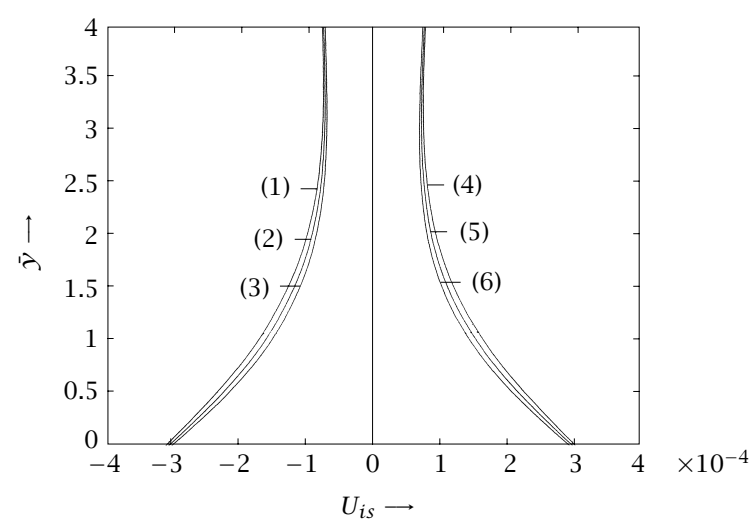

FIGURE 4.1. Induced steady axial velocity component of the fluid $U_{i s}$ in the boundary layer for $R=500, \epsilon=0.05$. (1) $X=-1, \lambda=0.01$, (2) $X=-1$, $\lambda=0.16$, (3) $X=-1, \lambda=0.31$, (4) $X=1, \lambda=0.01$, (5) $X=1, \lambda=0.16$, (6) $X=1, \lambda=0.31$.

4. Results and discussion. We have seen that the third- and fourth-order solutions consist of the steady part in addition to the periodic one. As the contribution of steady term in the fourth-order is more significant to the solution, we take up the fourth-order solution for discussion.

We observe that the normal oscillation of the wall causes at first the periodic flow in the boundary layer having the same frequency as that of the wall oscillation and then it causes the flows of higher harmonics in the boundary layer and induces periodic flow in the outer flow successively. The components of the velocity of the fluid both for outer and inner flow have been plotted against $y$ and $\bar{y}$ in Figures 4.1 to 4.13, respectively, for various values of $x$ and $t$, taking $R=500, \varepsilon=0.05$.

The inner steady streaming parts of both fluid and dust are plotted against $\bar{y}$ for various concentration parameter $\lambda$ (Figures 4.1 and 4.2). We find that both in the case of fluid and dust the inner steady streaming parts approach a constant value in the form of the oscillation with respect to the distance from the wall.

The various values of the dust parameter $\lambda$ make the velocity profiles of both the fluid and the dust well separated for small values of $t$. For a given $x$, they approach a constant value as $y$ increases.

We observe from Figures 4.3 and 4.4 that the axial velocity components $U_{p^{o}}$ of the dust are less than $U_{o}$ of the fluid for negative values of the $x$. It is also seen that both $U_{o}$ and $U_{p^{o}}$ increase as $y$ increases. The increase in the value of the concentration parameter $\lambda$ results in the increase of velocity components. But it is interesting to note that both $U_{o}$ and $U_{p^{o}}$ are becoming steady as $y$ increases further and approach almost equal values. More important the dust parameter $\lambda$ has got more impact on the motion of the fluid than on the dust.

Next we can observe the nature of the transverse velocity components $V_{o}$ and $V_{p^{o}}$ of the fluid and the particles of the outer flow from Figures 4.5, 4.6, and 4.7, respectively. Both $V_{o}$ and $V_{p^{o}}$ become steady as $y$ increases. We note that the impact of the dust parameter $\lambda$ is felt more on the motion of the fluid than on the dust even for small values of $t$. 


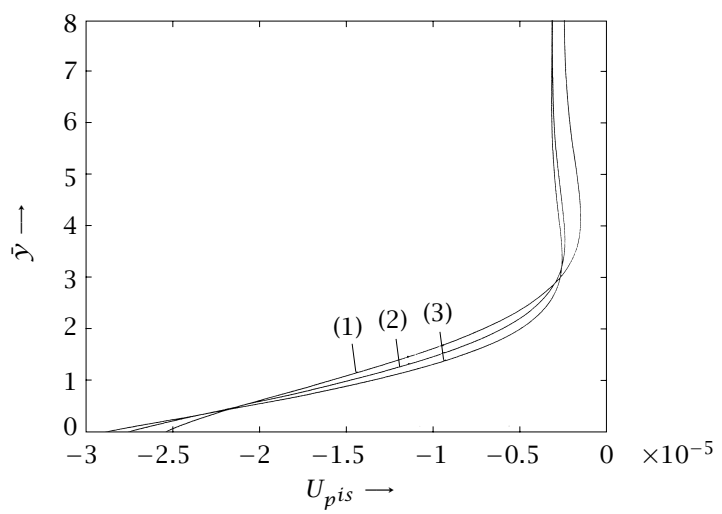

FIGURE 4.2. Induced steady axial velocity component of the particle $U_{p}$ is in the boundary layer for $R=500, \epsilon=0.05$. (1) $X=-0.1, \lambda=0.01$, (2) $X=-0.1$, $\lambda=0.36$, (3) $X=-0.1, \lambda=0.71$.

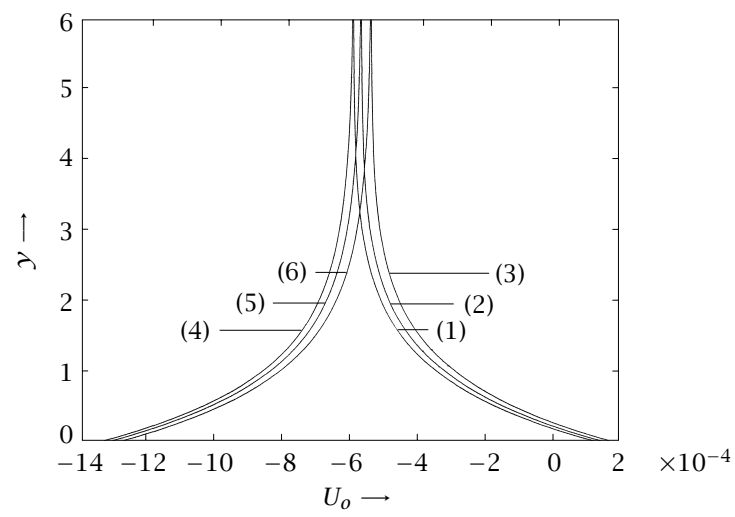

FIgURE 4.3. Outer flow axial velocity component of fluid $U_{o}$ for $R=500$, $\epsilon=0.05, t=2$. (1) $X=-1, \lambda=0.01$, (2) $X=-1, \lambda=0.16$, (3) $X=-1$, $\lambda=0.31$, (4) $X=1, \lambda=0.01$, (5) $X=1, \lambda=0.16$, (6) $X=1, \lambda=0.31$.

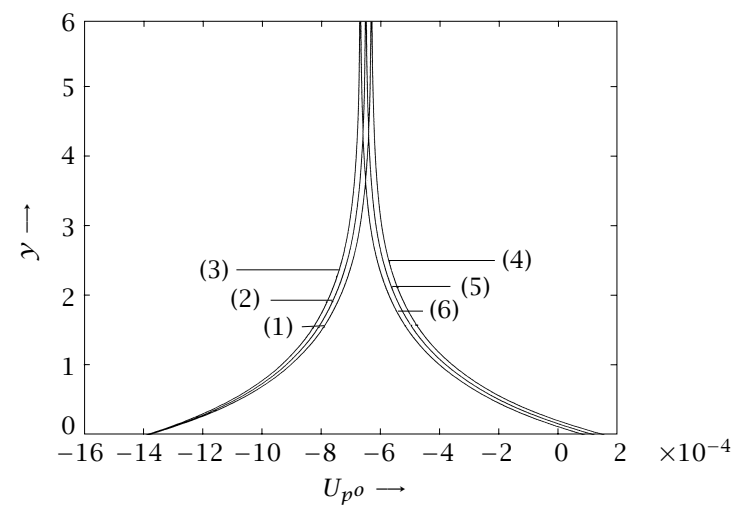

FIGURE 4.4. Outer flow axial velocity component of particle $U_{p^{o}}$ for $R=500$, $\epsilon=0.05, t=1$. (1) $X=-1, \lambda=0.01$, (2) $X=-1, \lambda=0.16$, (3) $X=-1, \lambda=0.31$, (4) $X=1, \lambda=0.01$, (5) $X=1, \lambda=0.16$, (6) $X=1, \lambda=0.31$. 


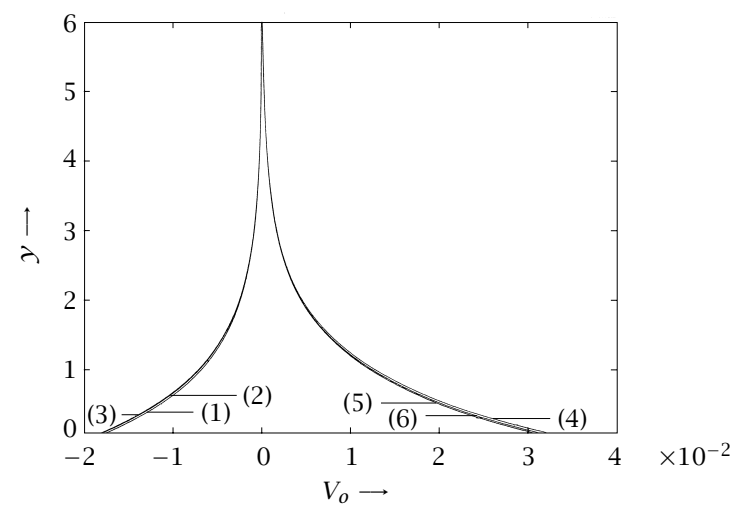

FIGURE 4.5. Outer flow transverse velocity component of fluid $V_{o}$ for $R=$ 500, $\epsilon=0.05, t=1$. (1) $X=-1, \lambda=0.01$, (2) $X=-1, \lambda=0.16$, (3) $X=-1$, $\lambda=0.31$, (4) $X=1, \lambda=0.01$, (5) $X=1, \lambda=0.16$, (6) $X=1, \lambda=0.31$.

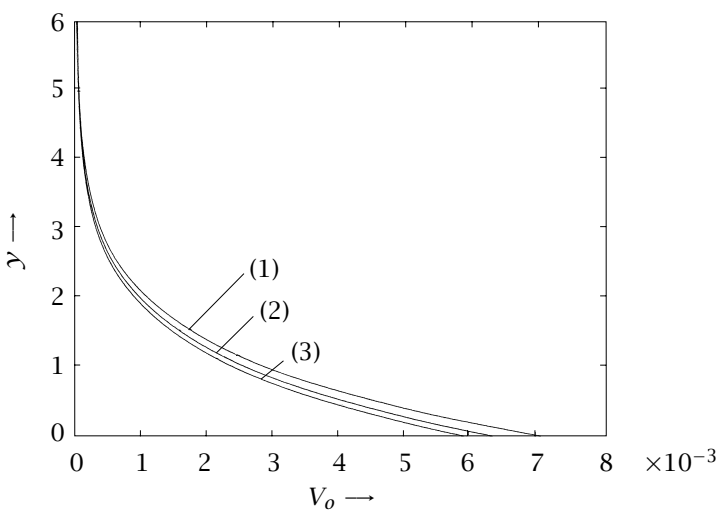

FIGURE 4.6. Outer flow transverse velocity component of fluid $V_{o}$ for $R=$ 500, $\epsilon=0.05, t=1.5$. (1) $X=-1, \lambda=0.01$, (2) $X=-1, \lambda=0.36$, (3) $X=-1$, $\lambda=0.71$.

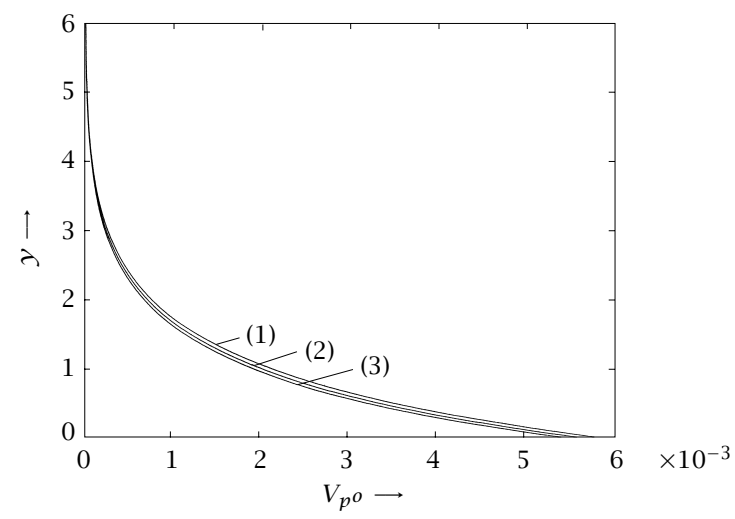

FigURE 4.7. Outer flow transverse velocity component of particle $V_{p^{o}}$ for $R=500, \epsilon=0.05, t=2$. (1) $X=-1, \lambda=0.01$, (2) $X=-1, \lambda=0.16$, (3) $X=-1, \lambda=0.31$. 


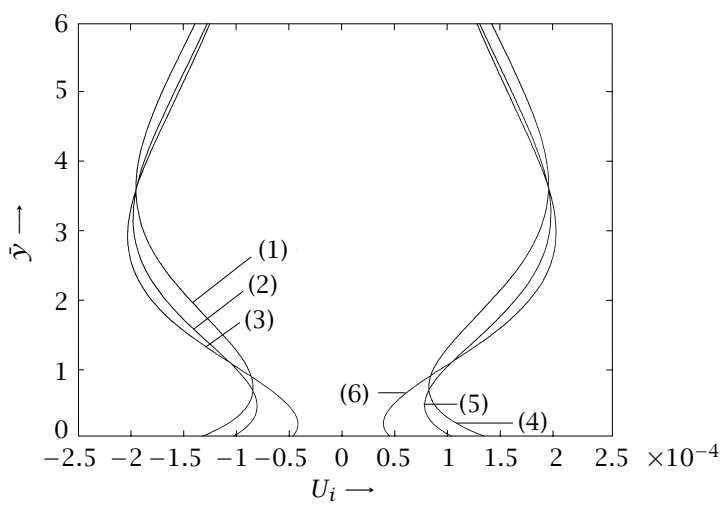

FIGURE 4.8. Axial velocity component of the fluid $U_{i}$ in the boundary layer for $R=500, \epsilon=0.05, t=1$. (1) $X=-0.5, \lambda=0.01$, (2) $X=-0.5, \lambda=0.16$, (3) $X=-0.5, \lambda=0.31$, (4) $X=0.5, \lambda=0.01$, (5) $X=0.5, \lambda=0.16$, (6) $X=0.5$, $\lambda=0.31$.

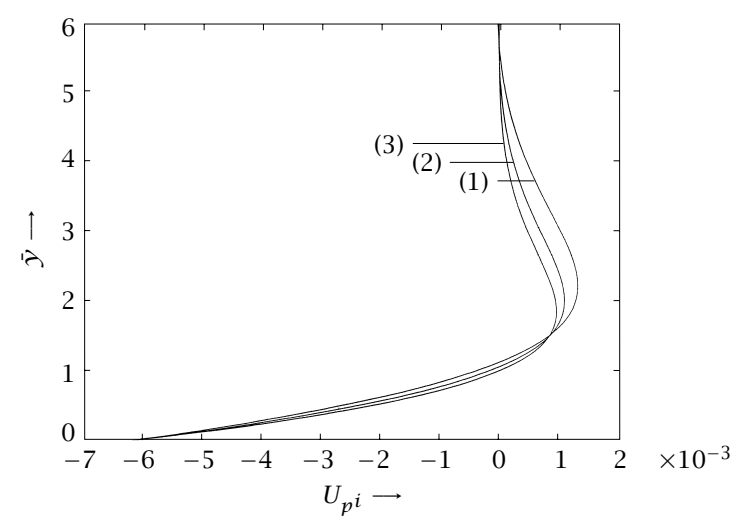

FiguRE 4.9. Axial velocity component of the particle $U_{p^{i}}$ in the boundary layer for $R=500, \epsilon=0.05, t=2$. (1) $X=0.5, \lambda=0.01$, (2) $X=0.5, \lambda=0.36$, (3) $X=0.5, \lambda=0.71$.

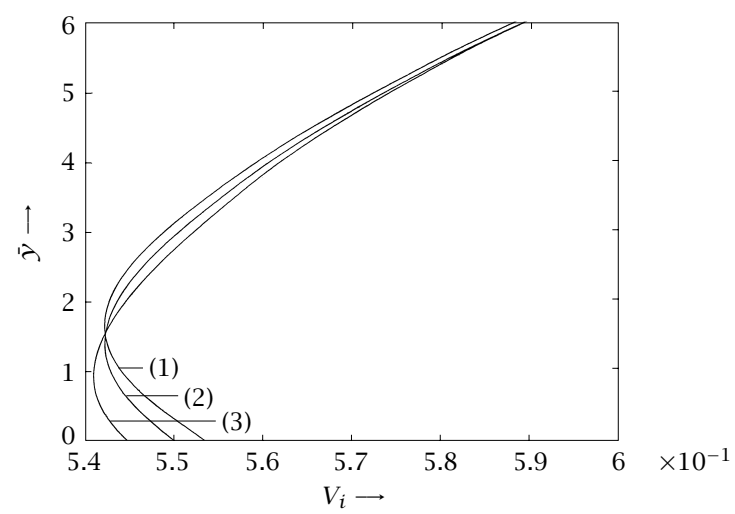

FIGURE 4.10. Transverse velocity component of the fluid $V_{i}$ in the boundary layer for $R=500, \epsilon=0.05, t=1$. (1) $X=-0.5, \lambda=0.01$, (2) $X=-0.5$, $\lambda=0.36$, (3) $X=-0.5, \lambda=0.71$. 


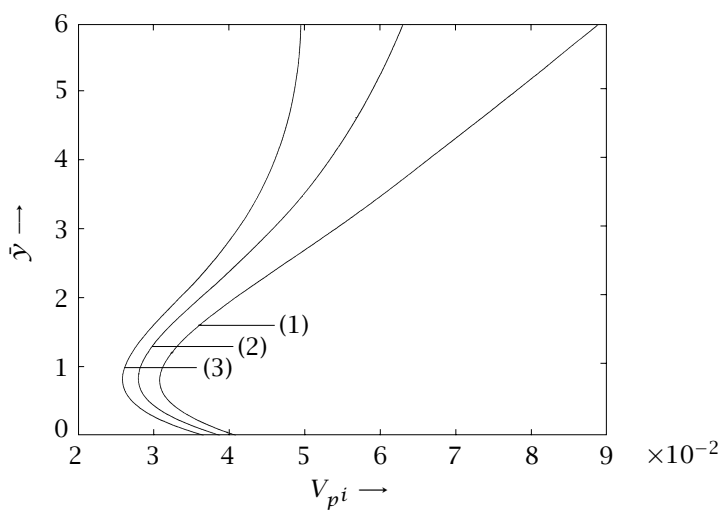

FIGURE 4.11. Transverse velocity component of the particle $V_{p^{i}}$ in the boundary layer for $R=500, \epsilon=0.05, t=2$. (1) $X=-1, \lambda=0.01$, (2) $X=-1$, $\lambda=0.36$, (3) $X=-1, \lambda=0.71$.

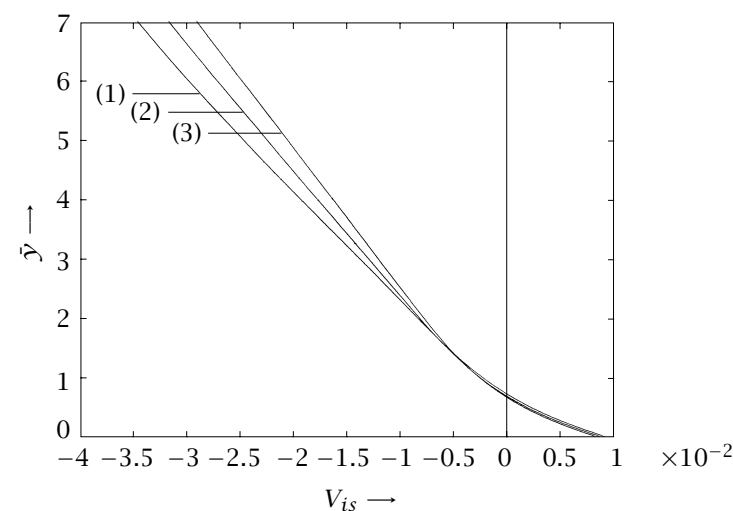

FIGURE 4.12. Induced steady transverse velocity component of the fluid $V_{i s}$ in the boundary layer for $R=500, \epsilon=0.05$. (1) $X=1, \lambda=0.01$, (2) $X=1$, $\lambda=0.36$, (3) $X=1, \lambda=0.71$.

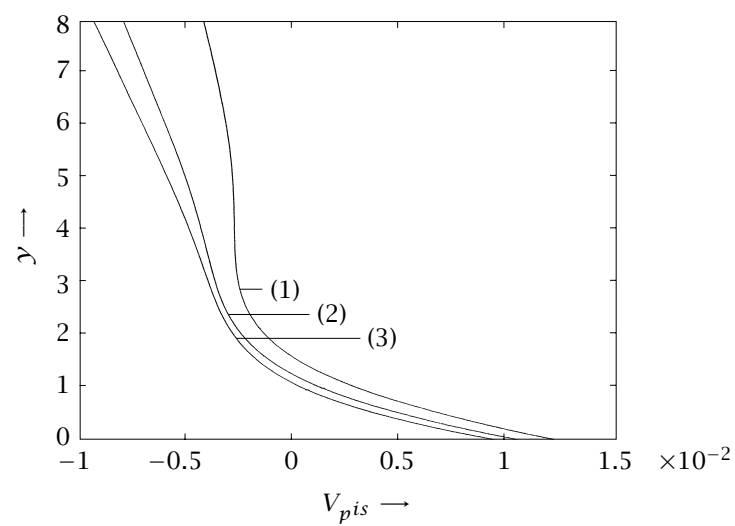

FIGURE 4.13. Induced steady transverse velocity component of the particle $V_{p i s}$ in the boundary layer for $R=500, \epsilon=0.05$. (1) $X=-1, \lambda=0.01$, (2) $X=-1, \lambda=0.36$, (3) $X=-1, \lambda=0.71$. 
The behaviour of velocity components $U_{i}$ and $U_{p^{i}}$ of the fluid at dust, can be studied from Figures 4.8 and 4.9, respectively. Here we see that $U_{i}$ is more oscillatory than $U_{p^{i}}$. But both become steady as $y$ increases. The separation of profiles is felt more in a particular range of values of $y$. The presence of dust seems to have more impact on the fluid than on the dust as seen from the oscillatory nature of the fluid motion.

We study $V_{i}$ and $V_{p^{i}}$ from Figures 4.10 and 4.11. It is very much interesting to note that with the increase of $y$ while the dust parameter $\lambda$ makes the profiles of the dust more separated it makes the profiles of the fluid more closer.

From Figures 4.12 and 4.13 we study the behaviour of inner transverse steady velocity components $V_{i s}$ and $V_{p^{i s}}$ of the fluid and the dust. Unlike in other cases the presence of dust has got more impact on the motion of the dust than on the fluid.

\section{REFERENCES}

[1] J. C. Burns and T. Parkes, Peristaltic motion, J. Fluid Mech. 29 (1967), 731-734.

[2] H. Dhar and K. D. Nanda, Induced flow due to normal oscillation of a wavy wall, Bull. Calcutta Math. Soc. 81 (1989), no. 5, 382-393. MR 91a:76028. Zbl 687.76032.

[3] J. Kevorkian and J. D. Cole, Perturbation Methods in Applied Mathematics, Applied Mathematical Sciences, vol. 34, Springer-Verlag, New York, 1981. MR 82g:34082. Zbl 456.34001 .

[4] V. Ramamurthy and U. S. Rao, Flow of a dusty fluid due to wavy motion of a wall for moderately large Reynolds numbers, Int. J. Math. Math. Sci. 12 (1989), no. 3, 559578. Zbl 674.76095.

[5] K. Tanaka, Induced flow due to wavy motion of a wall. I. The case of small and moderately large Reynolds numbers, J. Phys. Soc. Japan 42 (1977), no. 1, 297-305. MR 58\#32370.

[6] G. Taylor, Analysis of the swimming of microscopic organisms, Proc. Roy. Soc. London Ser. A 209 (1951), 447-461. MR 13,596a. Zbl 043.40302.

[7] F. C. P. Yin and Y. C. Fung, Comparison of theory and experiment in peristaltic transport, J. Fluid Mech. 47 (1971), 93-112.

K. Kannan: Department of Mathematics, Shanmugha College of Engineering, THIRUMALAISAMUDRAM, THANJAVUR-613402, TAMIL NADU, INDIA

E-mail address: kannan@maths.sce.ac. in

V. Ramamurthy: Department of Mathematics, A. C College of Technology, KARAIKUDAI, TAMIL NADU, INDIA 


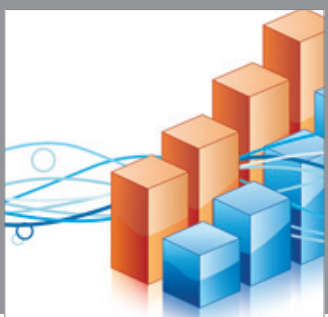

Advances in

Operations Research

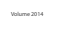

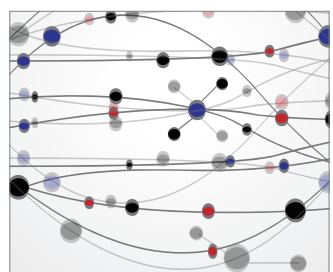

\section{The Scientific} World Journal
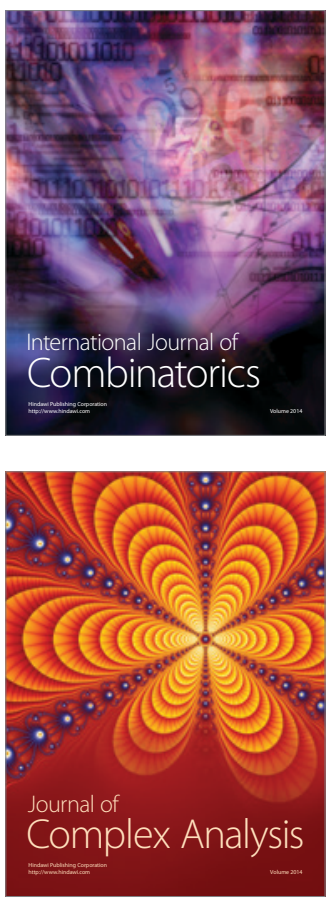

International Journal of

Mathematics and

Mathematical

Sciences
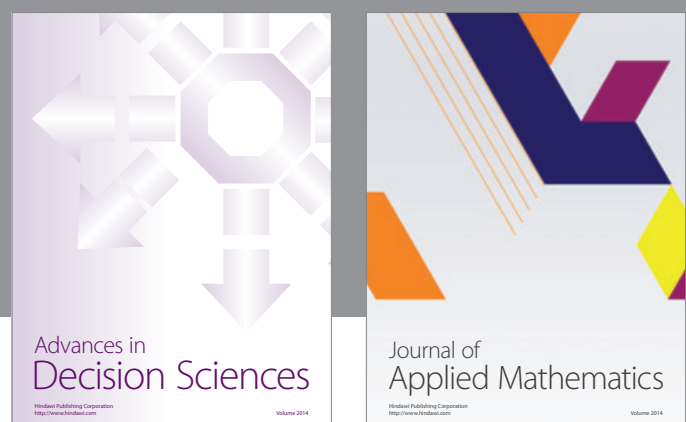

Journal of

Applied Mathematics
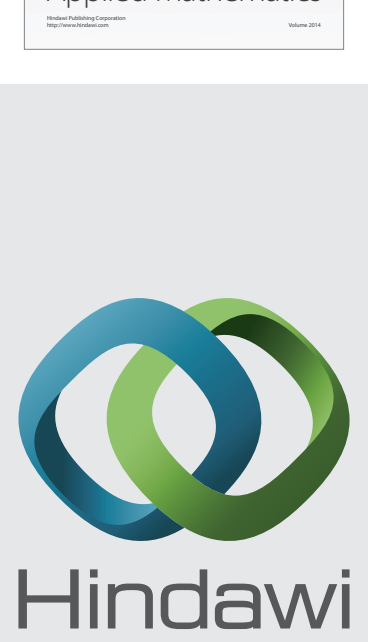

Submit your manuscripts at http://www.hindawi.com
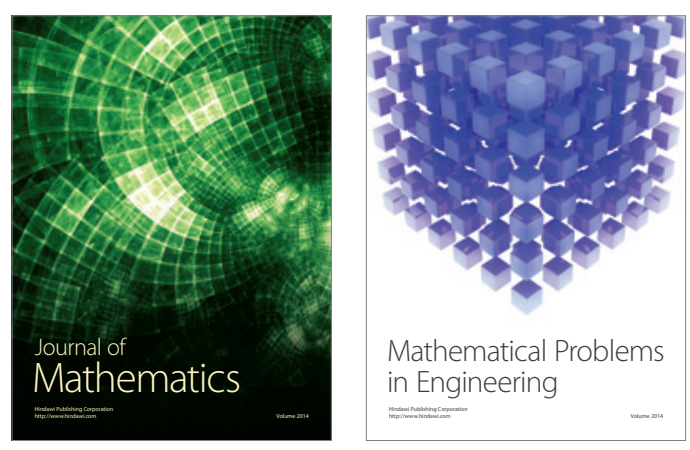

Mathematical Problems in Engineering
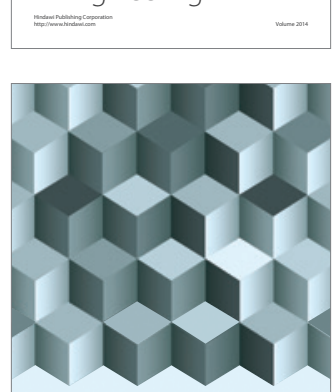

Journal of

Function Spaces
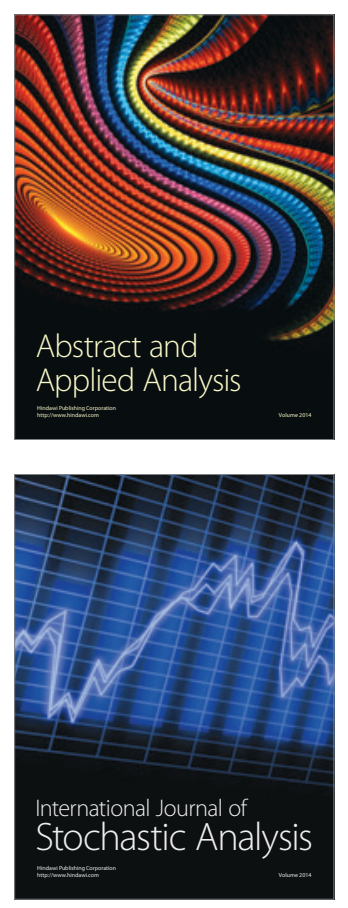

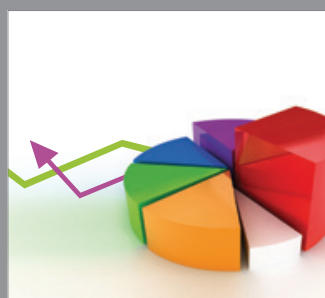

ournal of

Probability and Statistics

Promensencen
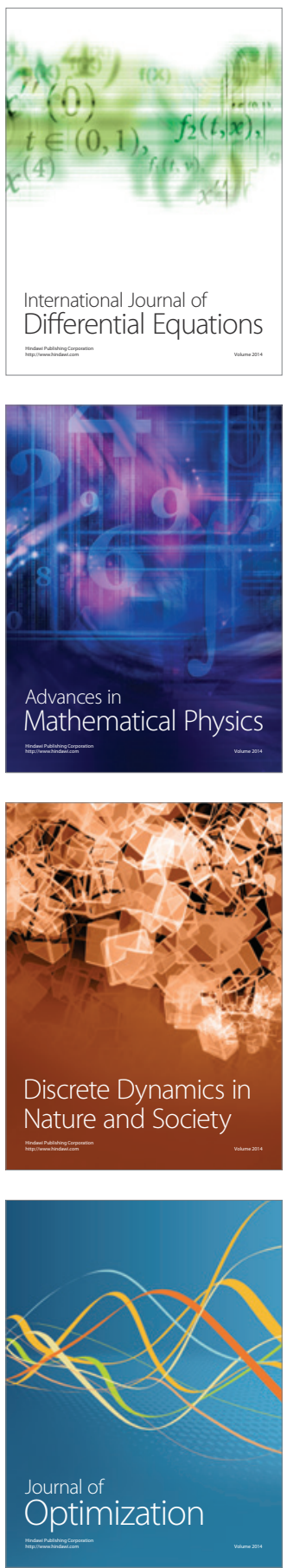